\title{
An overview of the experimental study of quark-gluon matter in high-energy nucleus-nucleus collisions
}

\author{
Anton Andronic* \\ Reasearch Division and EMMI, GSI Helmholtzzentrum für \\ Schwerionenforschung, D-64291 Darmstadt, Germany
}

\begin{abstract}
An overview is given on the experimental study of quark-gluon matter produced in relativistic nucleus-nucleus collisions, with emphasis on recent measurements at the Large Hadron Collider.
\end{abstract}

\section{Introduction}

The goal of high-energy nucleus-nucleus collisions is to produce and characterize a state of nuclear (Quantum Chromo-Dynamics) matter at (energy) densities well above the nuclear ground state $\left(\varepsilon_{0} \simeq 0.15 \mathrm{GeV} / \mathrm{fm}^{3}\right)$, an idea now 40 years old [1]. At high densities and/or at high temperatures one expects [2 4] that quarks, the building blocks of hadrons, are no longer confined but move freely over distances larger than the size of the nucleon (about $1 \mathrm{fm}=10^{-15} \mathrm{~m}$ ). Such a deconfined state of matter, earlier named the Quark-Gluon Plasma (QGP) [5], was the state of the Universe within the first (about 10) microseconds of its creation in the Big Bang [6] and may exist as well in the core of neutron stars [7] (see Ref. [4] for an earlier discussion). The characterization of quark-gluon matter in terms of its equation of state (EoS, relating pressure to energy) and of its transport properties (viscosity, diffusion coefficients) and delineating its phase diagram [8] is a major ongoing research effort 9 13.

At low energies (beam energies per nucleon of up to $10 \mathrm{GeV} / A$ on a fixed target, corresponding to center of mass energies per nucleon pair, $\sqrt{s_{\mathrm{NN}}} \lesssim 5 \mathrm{GeV}$ ) it is expected that compressed nucleonic matter is produced ("highly excited nuclear matter" [1]). The EoS of nuclear matter [14] at densities a few times the normal nuclear density $\left(\rho_{0}=0.17\right.$ $\mathrm{fm}^{-3}=2.7 \cdot 10^{14} \mathrm{~g} / \mathrm{cm}^{3}$ ), expressed as the nuclear compressibility, has relevance for the maximum mass of neutron stars (see Ref. [15] for an overview).

From lattice QCD calculations, a deconfinement phase transition for an energy density of about $1 \mathrm{GeV} / \mathrm{fm}^{3}$ was predicted (see Ref. [16] for an early review). It was shown [17] that the phase transition at zero baryochemical potential, $\mu_{B}$, is of crossover type, namely with a continuous, smooth, increase of thermodynamic quantities. The value of the (pseudo-)critical temperature, $T_{c}$, at vanishing baryochemical potential $\left(\mu_{B}\right)$ is currently calculated in lattice QCD [18,19] to be in the range 155-160 MeV. The existence of a

*A.Andronic@gsi.de 
critical point, denoting the end of the first order phase transition line, a point where the phase transition is of a second order, is a fundamental question, addressed both experimentally [20] and theoretically [21,22] (see a review in Ref. [23]).

A nucleus-nucleus collision is a highly-dynamical event. One can identify, schematically, the following stages: i) initial collisions, occuring during the passage time of the nuclei $\left(t_{\text {pass }}=2 R / \gamma_{\mathrm{cm}} c\right)$; ii) thermalization: equilibrium is established; iii) expansion and cooling (in a deconfined state); iv) chemical freeze-out (possibly at hadronization): inelastic collisions cease, hadron yields (and distribution over species) are frozen; v) kinetic freeze-out: elastic collisions cease, spectra and correlations are frozen.

The challenge is to characterize the hot (deconfined) stage iii), called early-on "fireball" [24], while most of the measurements are performed via hadrons (or their decay products) carrying primarily information from the system at stages iv) and v). Even though the early stage of hot deconfined matter remains inaccessible in a direct way because of quark confinement, there are experimental observables which carry precious information from this stage. Extracting the properties of the early hot (deconfined) stage is possible only via models. At low and intermediate energies hadronic transport models [14,25 27] are employed, while at high energies hydrodynamics [28, 29] is widely used (becoming a "standard model" for the theoretical understanding of high energy nucleusnucleus collisions [30]). Hybrid approaches, combining hydrodynamics and transport, are also employed 31.

Based on model comparison to data in a broad range of collision energies, one can extract the following ranges of the fireball characteristics (values are in the system of units where $\hbar=c=1$ commonly used in high-energy physics):

- Temperature: $T=100-500 \mathrm{MeV}$, or up to a million times the temperature at the center of the Sun $\left(1 \mathrm{MeV} \simeq 10^{10} \mathrm{~K}\right)$;

- Pressure: $P=100-300 \mathrm{MeV} / \mathrm{fm}^{3}\left(1 \mathrm{MeV} / \mathrm{fm}^{3} \simeq 10^{33} \mathrm{~Pa}\right)$;

- Density: $\rho=1-10 \cdot \rho_{0}$;

- Volume: several thousands of $\mathrm{fm}^{3}$;

- Duration: $10-20 \mathrm{fm} / c$ (or about $3-6 \cdot 10^{-23} \mathrm{~s}$ ).

The experimental "control parameters" are: a) the collision energy (per nucleon pair, $\sqrt{s_{\mathrm{NN}}}$ ) ; b) the centrality of the collision (or, alternatively, the size of the colliding nuclei). Centrality is obtained from specific measurements (see Ref. 32] for such measurements in ALICE). The usual way of expressing centrality is as percentage of the geometrical cross section; another way is via the number of participating nucleons, $N_{\text {part }}$, namely the nucleons involved in the creation of the fireball in the overlap region of the two colliding nuclei [24]; $N_{\text {part }}$ is calculated as an average over a given centrality range employing the Glauber model [33]. An illustration of the dependence of $N_{\text {part }}$ and $N_{\text {coll }}$ (the number of nucleon-nucleon collisions) on the centrality range is presented in Fig. 1 for the LHC energy (the inelastic nucleon-nucleon cross section, $\sigma_{\mathrm{NN}}^{\text {inel }}$, is an input for Glauber model calculations; for its energy dependence, see an overview in Ref. [34]).

After the initial measurements at the Bevalac (Berkeley), the program of heavy-ion collisions continued at higher energies at Brookhaven at the Alternating Gradient Synchrotron (AGS) and at CERN at the Super-Proton Synchrotron (SPS), while in the low 


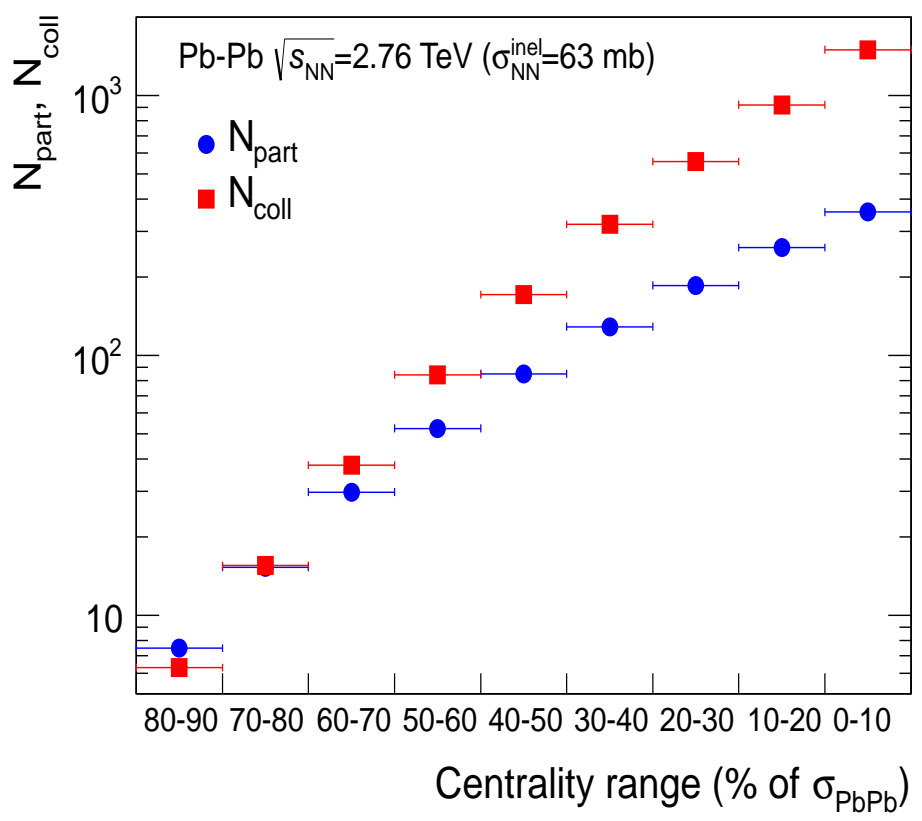

Figure 1: Centrality dependence of $N_{\text {part }}$ and $N_{\text {coll }}$ (average values) for $\mathrm{Pb}-\mathrm{Pb}$ collisions at $\sqrt{s_{\mathrm{NN}}}=2.76 \mathrm{TeV}$ (the total interaction cross section is $\sigma_{\mathrm{PbPb}}=7.7 \mathrm{~b}$ ).

energy range measurements were performed at GSI Darmstadt at the Schwerionensynchrotron (SIS). Started in year 2000, the experimental program at the Relativistic Heavy Ion Collider (RHIC) at Brookhaven spans $\sqrt{s_{\mathrm{NN}}}$ from about 8 to $200 \mathrm{GeV}$ (see, for earlier results, experimental summaries in [35 38] and an overview in [39]). The study of QCD matter has entered a new era in year 2010 with the advent of $\mathrm{Pb}-\mathrm{Pb}$ collisions at the Large Hadron Collider (LHC), delivering the largest ever collision energy, $\sqrt{s_{\mathrm{NN}}}=2.76$ $\mathrm{TeV}$, more than a factor of 10 larger than previously available. An overview of the first LHC data are available in Ref. [13, 40].

\section{Global observables}

Global (bulk) observables are employed to characterize the bulk thermal properties of the system. In Fig. 2 the collision energy dependence of the measured charged-particle rapidity density $\mathrm{d} N_{\mathrm{ch}} / \mathrm{d} y$ is shown. The data are for mid-rapidity, $y=0$ (where particles are emitted in the transverse direction); rapidity is defined as:

$$
y=\frac{1}{2} \ln \frac{E+p_{\mathrm{L}}}{E-p_{\mathrm{L}}}=\tanh ^{-1}\left(\beta_{\mathrm{L}}\right)
$$

with $p_{\mathrm{L}}\left(\beta_{\mathrm{L}}\right)$ the longitudinal momentum (velocity, in units of $c$ ), $E=\sqrt{m^{2}+p^{2}}$ the total energy.

The measurement of the charged hadrons pseudorapidity $(\eta=-\ln [\tan (\theta / 2)]$, with $\theta$ the polar angle) density, $\mathrm{d} N_{\mathrm{ch}} / \mathrm{d} \eta$, at the LHC was eagerly awaited and showed [41] that the increase compared to the measurement at RHIC is by a factor of about 2.2 for central collisions. Interpreted as the outcome of an increase of the initial entropy density, this increase can be translated into a factor of 1.3 increase of the initial temperature [42]. The ALICE measurement at the LHC confirmed the phenomenological $\left(\sqrt{s_{\mathrm{NN}}}\right)^{0.3}$ behavior seen 


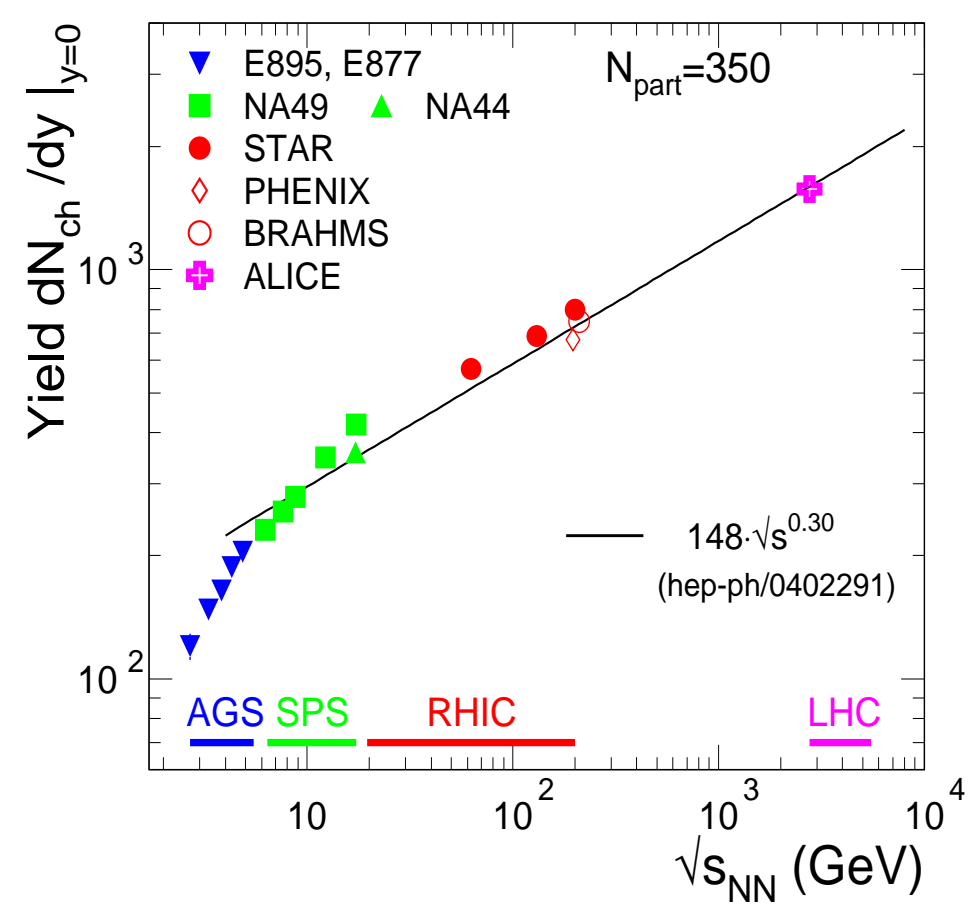

Figure 2: Collision energy dependence of charged-particle rapidity density $\mathrm{d} N_{\mathrm{ch}} / \mathrm{d} y$ (sum of pions, kaons and protons and their antiparticles) at midrapidity, measured by various experiments in central collisions corresponding to $N_{\text {part }}=350$.

at lower energies [43], see Fig. 2. The measurement at the LHC [41] clearly demonstrated that the increase of $\mathrm{d} N_{\mathrm{ch}} / \mathrm{d} \eta$ with energy is steeper in nucleus-nucleus (AA) collisions compared to pp collisions, where the functional form is $\left(\sqrt{s_{\mathrm{NN}}}\right)^{0.22}$. The data points shown in Fig. 22 (left panel) are obtained by summing the measured $\mathrm{d} N / \mathrm{d} y$ yields for pions, kaons and protons and their antiparticles, see below.

The centrality dependence of $\mathrm{d} N_{\mathrm{ch}} / \mathrm{d} \eta$ is at the LHC [44] identical to that measured at RHIC, see Fig. 3, pointing to a similar mechanism of particle production at the two energies. A model of the parton structure of matter at low parton fractional momentum $x$, the Color Glass Condensate [45, 46], describes well the data (see an extended comparison to theoretical models in Ref. [44]).

Utilizing, in addition to particle counting, the momentum measurement (or, alternatively, measuring the total hadron energy in calorimeters), see Fig. 4, one can extract from the data the energy density at the thermalization time. This involves a space-time model of the collision, which was originally put forward by Bjorken [48] and which is presently incorporated in a more realistic way in hydrodynamical models. In the Bjorken model, the energy density is:

$$
\varepsilon=\frac{1}{A_{\mathrm{T}}} \frac{\mathrm{d} E_{\mathrm{T}}}{\mathrm{d} y} \frac{1}{c \tau_{0}},
$$

where $E_{\mathrm{T}}$ is the measured transverse energy and $A_{T}=\pi R^{2}$ is the geometric transverse area of the fireball (for central $\mathrm{Pb}-\mathrm{Pb}$ collisions, $A_{T} \simeq 150 \mathrm{fm}^{2}$ ). Assuming a conservative value for the equilibration time, $\tau_{0}=1 \mathrm{fm} / c$ (a value which dates back to Bjorken [48]), one calculates for the LHC energy an energy density of $\varepsilon_{L H C}=14 \mathrm{GeV} / \mathrm{fm}^{3}$ [47]. The "threshold" value for deconfinement, of about $1 \mathrm{GeV} / \mathrm{fm}^{3}$, from lattice QCD calculations [16], is reached at $\sqrt{s_{\mathrm{NN}}} \simeq 5 \mathrm{GeV}$. Note that the equilibration time may depend on the 


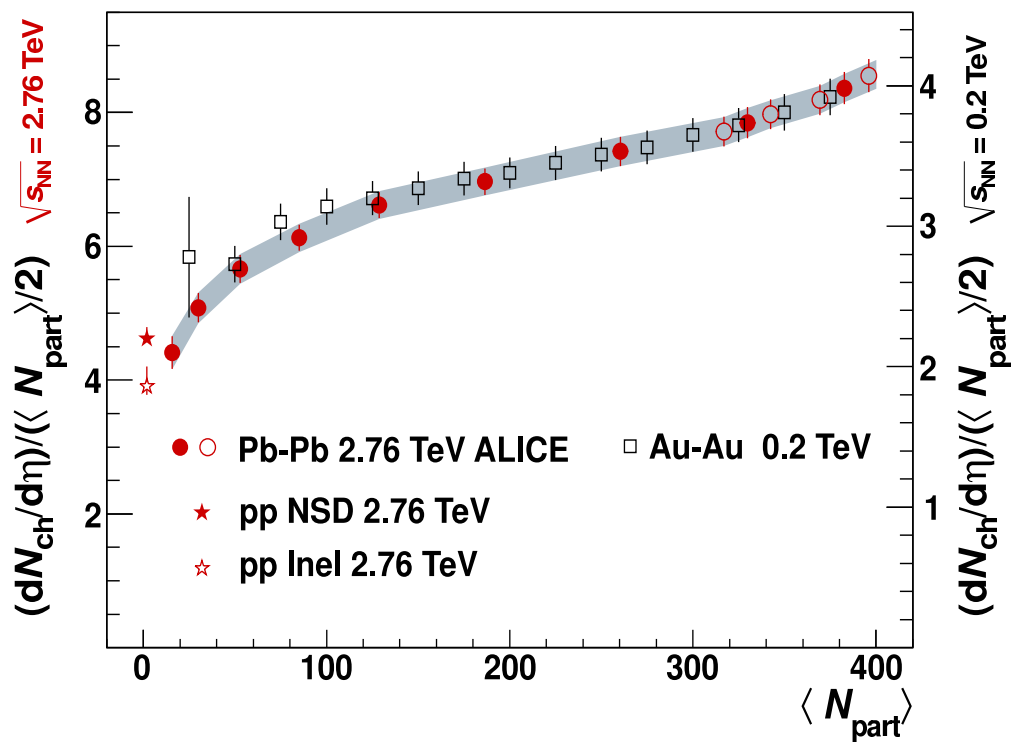

Figure 3: Centrality dependence of the charged particle pseudorapidity density in $\mathrm{Pb}-\mathrm{Pb}$ collisions at the LHC and in $\mathrm{Au}-\mathrm{Au}$ collisions at RHIC (plot from Ref. [44], note the right axis scale for the RHIC data).

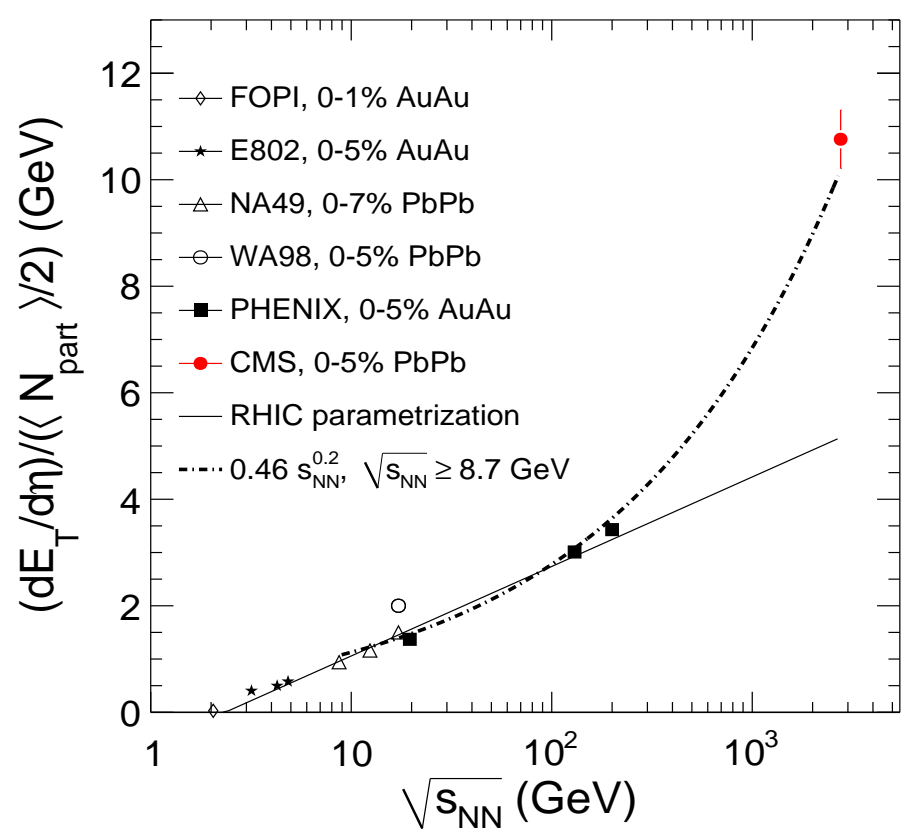

Figure 4: Collision energy dependence of the pseudorapidity density of transverse energy in central collisions (plot from Ref. [47]).

collision energy; values well below $1 \mathrm{fm} / c$ are currently used in hydrodynamical models. 


\section{Hadron yields and the chemical freeze-out}

In Fig. 7 the collision energy dependence of identified hadron yields at mid-rapidity is shown. This comprises measurements, spanning more than 20 years, by experiments at the AGS: E895 [49 51], E866/E917 [52,53], E891 [54]; the SPS: NA49 [55 58], NA44 [59], NA57 [60]; RHIC: STAR [61 65, BRAHMS [66], PHENIX [67]; and the LHC: ALICE [68 72 .

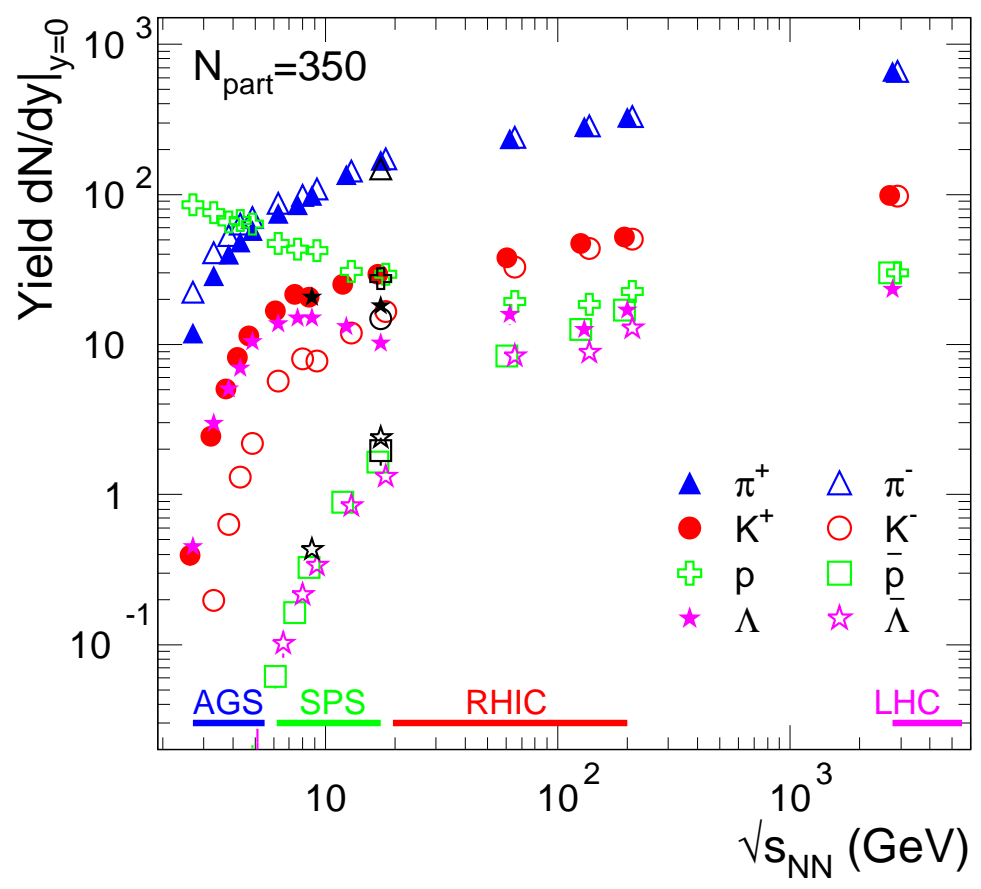

Figure 5: Collision energy dependence of the multiplicities (yield, $\mathrm{d} N / \mathrm{d} y$, at midrapidity) of pions, kaons, protons and $\Lambda$ hyperons and their antiparticles, measured in central collisions (corresponding to $N_{\text {part }}=350$ ) of $\mathrm{Au}$ or $\mathrm{Pb}$ nuclei.

The monotonic decrease of the proton yield as a function of energy indicates that fewer and fewer of the nucleons (or their valence $u, d$ quarks) in the colliding nuclei are "stopped" in the fireball. An onset of meson production is seen, with the kaons (heavier and containing a strange quark) produced less abundantly than pions. The difference in production yields of $\pi^{+}$and $\pi^{-}$at low energies reflects the isospin composition of the fireball. The difference between $K^{+}$and $K^{-}$meson yields and $\Lambda$ and $\bar{\Lambda}$ hyperon production is determined by the quark content of the hadrons, $K^{+}(u \bar{s}), K^{-}(\bar{u} s) \Lambda(u d s), \bar{\Lambda}(\bar{u} \bar{d} \bar{s})$. The availability in the fireball of valence $u, d$ quarks from colliding nucleons "stopped" in the fireball leads to a preferential production of hadrons carrying those quarks. These differences vanish gradually for higher energies, where the hadrons are mostly newly created (reflecting Einstein's famous equation $m=E / c^{2}$ ) and the production yields exhibit a clear mass ordering.

Good fits of the measurements are achieved with the thermal model [74] with 3 parameters: temperature $T$, baryochemical potential $\mu_{B}$, and volume $V$, see Fig. 6 for the fit of data at the LHC [73]. Remarkably, multiply-strange hyperons and (hyper)nuclei are well described by the model, which also explains [75] the equal production of matter and antimatter at the LHC [68]. An interesting question remains whether at hadronization the 


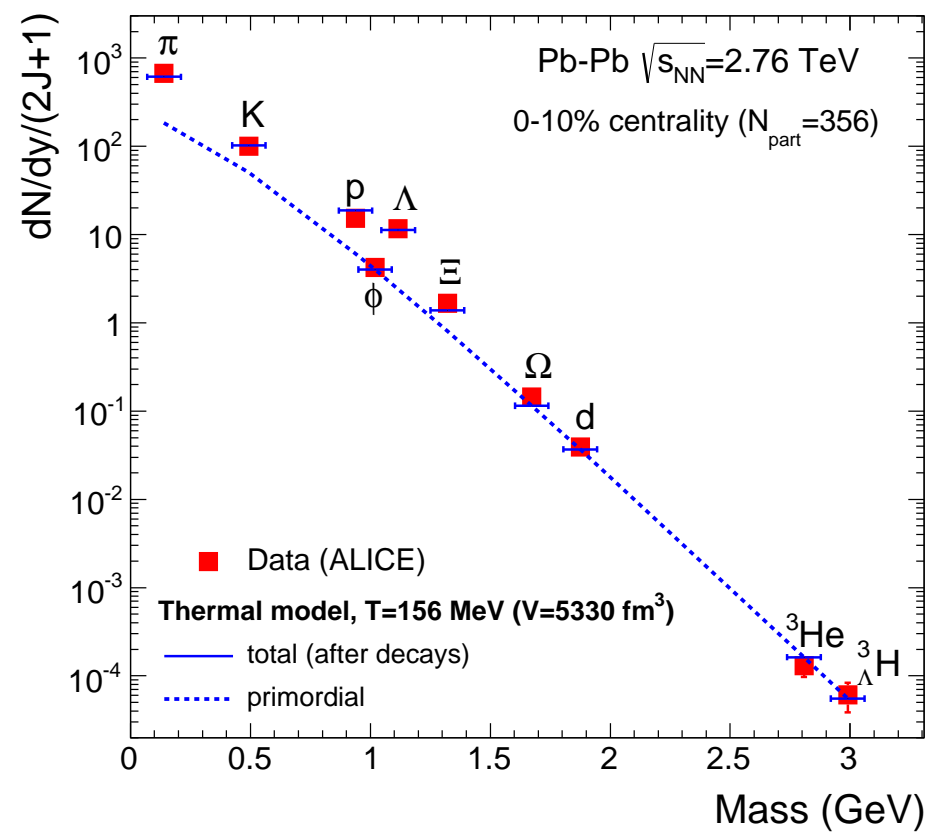

Figure 6: Measured hadron abundances divided by the spin degeneracy factor $(2 J+1)$ in comparison with thermal model calculations for the best fit to data [73] in central $\mathrm{Pb}-\mathrm{Pb}$ collisions at the LHC. For the model, plotted are the "total" yields, including all contributions from high-mass resonances (for the $\Lambda$ hyperon, the contribution from the electromagnetic decay $\Sigma^{0} \rightarrow \Lambda \gamma$, which cannot be resolved experimentally, is also included), and the ("primordial") yields prior to decays.

(hyper)nuclei are droplets of quark matter [76] or if they form via nucleon (and hyperon) coalescence.

The thermal model describes a snapshot of the collision, namely the chemical freezeout, which is assumed to be quasi-instantaneous. It provides a phenomenological link of data to the QCD phase diagram, a link identified early on [3,81] and discussed extensively more recently [78, 82,86].

The phenomenological phase diagram is shown in Fig. 7. Each point corresponds to a fit of hadron yields in central $\mathrm{Au}-\mathrm{Au}$ or $\mathrm{Pb}-\mathrm{Pb}$ collisions at a given collision energy. The agreement between the results from several independent analyses [64,78, 80] is remarkable. Note that in some cases [64,79,80] an additional fit parameter, the strangeness suppression factor $\gamma_{s}$, is used to test possible departure from equilibrium of hadrons containing strange quark(s). Values of $\gamma_{s}$ (slightly) below unity are found. An approach with more nonequilibrium parameters is also employed [87, 88], with somewhat different conclusions. Fits considering a spread in $T$ and $\mu_{B}$ were also performed [89].

A remarkable outcome of these fits is that $T$ increases with increasing energy (decreasing $\mu_{B}$ ) from about $50 \mathrm{MeV}$ to about $160 \mathrm{MeV}$, where it exhibits a saturation for $\mu_{B} \lesssim 300$ $\mathrm{MeV}$. This saturation of $T$ led to the connection to the QCD phase boundary, via the conjecture that the chemical freeze-out temperature can be the hadronization temperature [78] and that the two regimes in the phase diagram, Fig. 7, that of approximately constant $T$ for small $\mu_{B}$ values and of the strong increase of $T$ at large $\mu_{B}$, can imply the existence of a triple point in the QCD phase diagram [85] (see Ref. [90] for an earlier discussion). Various criteria for the chemical freeze-out were proposed [91,92]. 


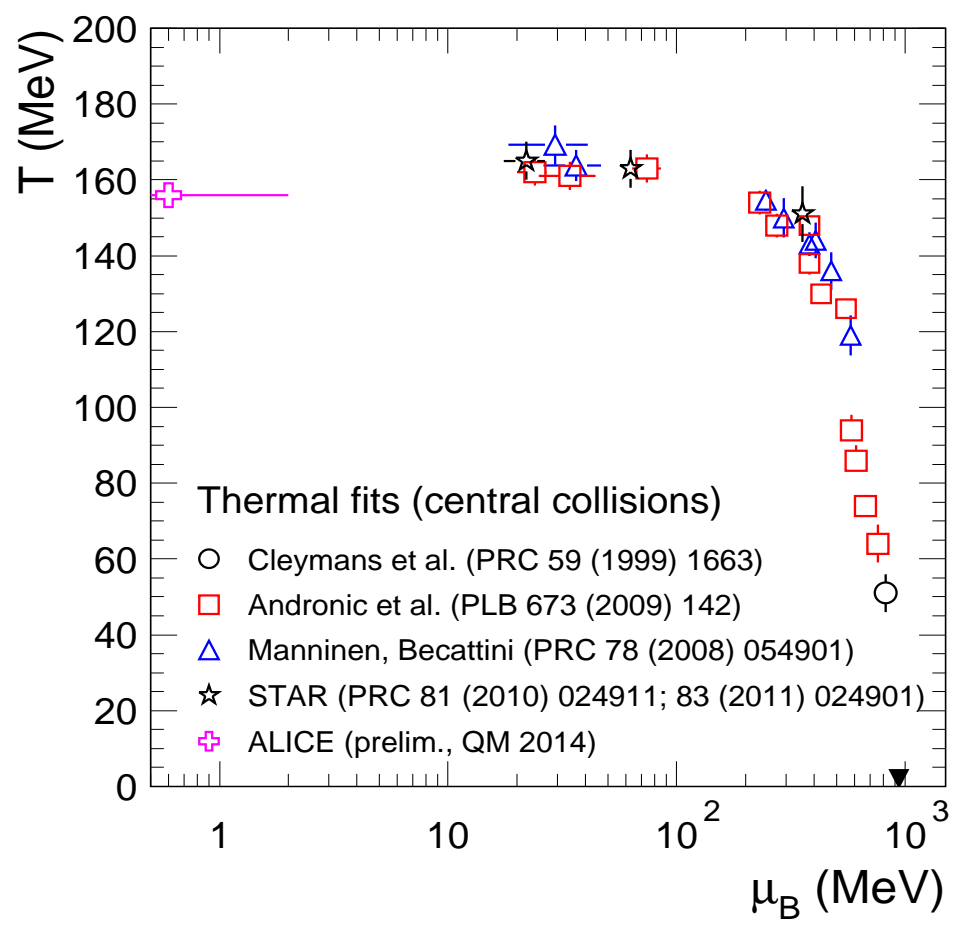

Figure 7: The phase diagram of strongly interacting matter with the points representing the thermal fits of hadron yields at various collision energies [64,73, 77, 80]. For the LHC, $\mu_{B}=0$ is the outcome of the fit, $0.6 \mathrm{MeV}$ is used here for the sake of proper representation with the logarithmic scale. The down-pointing triangle indicates ground state nuclear matter (atomic nuclei).

The thermal model provides the following values of thermodynamical quantities at chemical freeze-out (for high energies, corresponding to the "limiting temperature" $T_{\text {lim }}=$ $159 \mathrm{MeV}$; see Ref. 93] for the energy dependence): pressure $P \simeq 60 \mathrm{MeV} / \mathrm{fm}^{3}$, energy density $\varepsilon \simeq 330 \mathrm{MeV} / \mathrm{fm}^{3}$, entropy density $s=2.4 \mathrm{fm}^{-3}$, meson density $n=0.26 \mathrm{fm}^{-3}$, baryon density $n_{B}=0.06 \mathrm{fm}^{-3}$ (particles and antiparticles). In Fig. 8 an illustration is shown of the success of the thermal model in reproducing over a broad energy range the ratio of production of various hadron species. The calculations are performed with parametrizations for $T$ and $\mu_{B}$ as in Ref. [78. The non-monotonic dependence on energy of the $K^{+} / \pi^{+}$yield ratio was proposed as a signature [94], and the measurement taken as an evidence [56], for an onset of deconfinement, but can be understood well within the hadronic picture of the thermal model [78], as shown in Fig. 8 (right panel). Based on this success, the thermal model predictions provide a reliable guidance for experimental searches for other exotic nuclei 95 .

Current theoretical research addresses the question of flavor-dependent freeze-out [96, 97]; the role of interactions after chemical freeze-out [98] (in the hybrid model of Ref. [98] higher $T$ values are obtained for the LHC case); the effect of the extension of hadronic resonance spectrum beyond the currently established hadron states [99, 100]. The connection to fits in $\mathrm{e}^{+} \mathrm{e}^{-}$(see Ref. [101] and references therein) and in elementary hadronic collisions 102 remains also to be better understood.

Another sector of investigations concerns moments of net-electric charge [103] and net-proton [104] event-by-event multiplicity destributions. Such measurements have the 

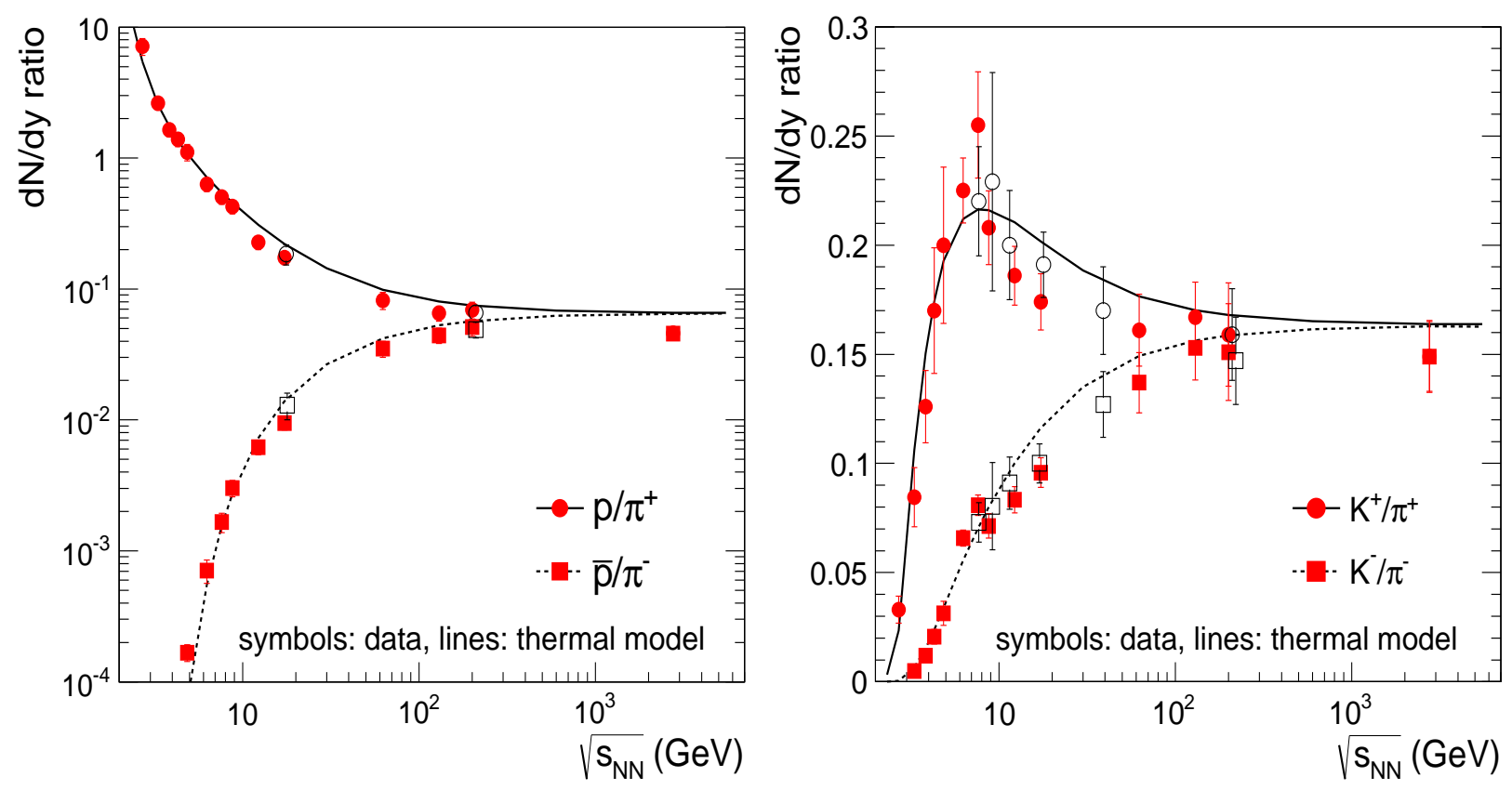

Figure 8: Collision energy dependence of ratios of yields of protons and antiprotons (left panel) and of kaons (right panel) to yields of pions. The symbols are data, the lines are thermal model calculations for energy-dependent parametrizations of $T$ and $\mu_{B}$ (as in Ref. [78]).

potential to reveal the critical point in the QCD phase diagram [90,105] (see also Ref. [22]). Fluctuations of baryon number and electric charge (determined essentially by the light quarks) can be calculated in lattice QCD, allowing an alternative way to determine $T$ and $\mu_{B}$ [106]. Currently, it appears that $T$ values extracted in this way [107] are somewhat lower that those of the fits of hadron yields.

\section{Collective flow and the kinetic freeze-out}

Collective flow is a distinct feature of nucleus-nucleus collisions, first observed in collisions at low energies at the Bevalac [108]. In central collisions one investigates the so-called radial flow, which is quantified fitting transverse momentum $\left(p_{\mathrm{T}}\right)$ spectra with the so-called "blast wave" model [109], obtaining in a convenient (albeit simplified) way bulk properties of the fireball at kinetic freeze-out. The extracted fit parameters, the temperature and the average transverse velocity $\left\langle\beta_{\mathrm{T}}\right\rangle$, are shown in Fig. 9 as a function of the collision energy. The measurements are by experiments EOS [110], FOPI [111], NA49 [112], STAR [61,64], and ALICE [68].

A strong increase of both $T$ and $\left\langle\beta_{\mathrm{T}}\right\rangle$ is seen at low energies (beam energies of up to $1 \mathrm{GeV} / A$ on fixed target). For $\sqrt{s_{\mathrm{NN}}} \gtrsim 5 \mathrm{GeV}$, a small further increase of $\left\langle\beta_{\mathrm{T}}\right\rangle$ is seen, reaching at the $\mathrm{LHC}\left\langle\beta_{\mathrm{T}}\right\rangle \simeq 0.65 c$ 68, and a constant kinetic freeze-out temperature, which is $50-60 \mathrm{MeV}$ lower than the chemical freeze-out value. At lower energies, the chemical freeze-out temperature is smaller than the kinetic one, which is unphysical and awaits a resolution.

In non-central collisions elliptic flow arises as a result of the initial (elliptic) transverse shape of the overlap zone of the two nuclei (participant eccentricity $\varepsilon_{\text {part }}$ ). Through the 


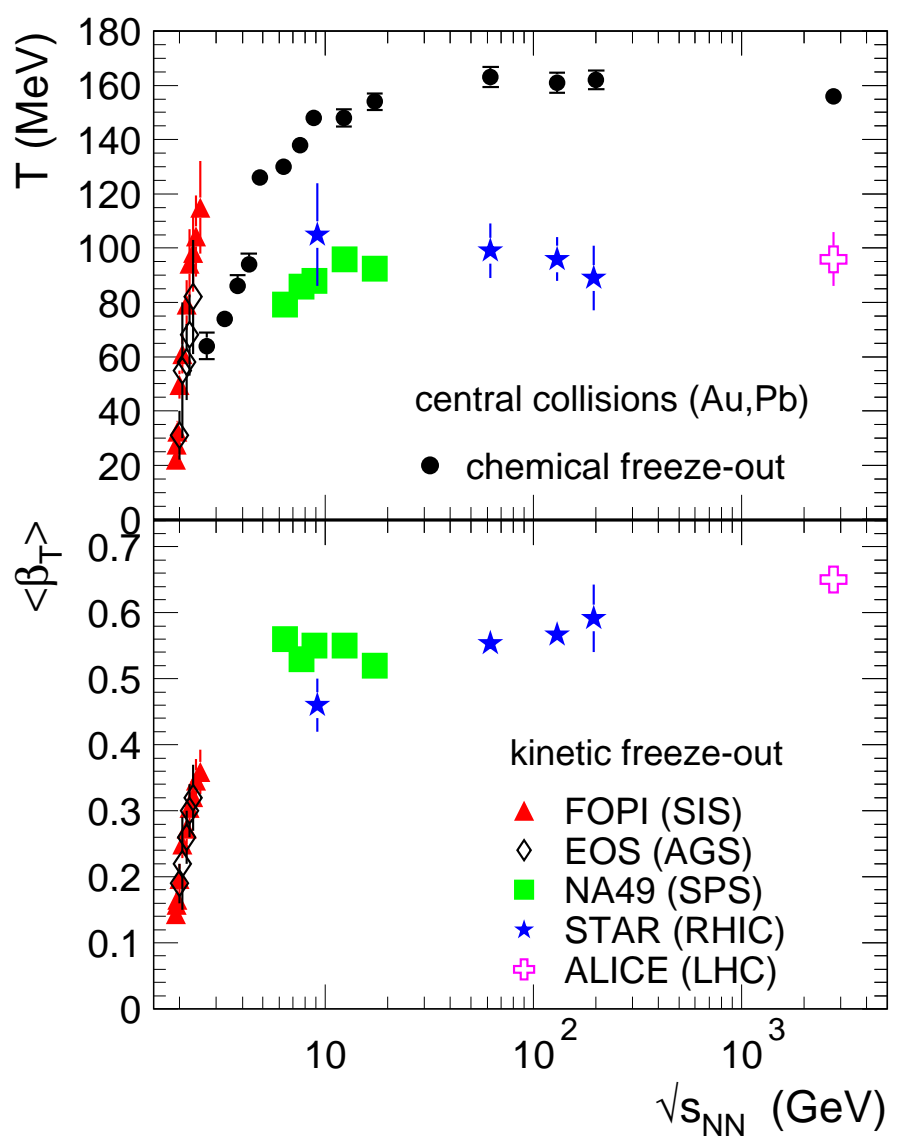

Figure 9: Collision energy dependence of collective radial flow in central collisions, quantified by the temperature $T$ and average velocity $\left\langle\beta_{\mathrm{T}}\right\rangle$ at kinetic freeze-out; the temperature at chemical freeze-out is represented by the dots.

initial gradients of the energy density (or pressure), this leads to an anisotropic angular emission of hadrons. This is quantified by the second order (quadrupole) Fourier coefficient $v_{2}=\langle\cos (2 \varphi)\rangle$, where $\varphi$ is the azimuthal angle with respect to the reaction plane. In Fig. 10 we show the energy dependence of elliptic flow, measured in mid-central collisions $\left(\left\langle N_{\text {part }}\right\rangle \simeq 160\right.$, corresponding to an average impact parameter value of $\left.\langle b\rangle \simeq 7 \mathrm{fm}\right)$ by experiments FOPI [113], E895 [114], E877 [115], CERES [116], NA49 [117, STAR [118], PHOBOS [119], ALICE [120], and CMS [121]. The complex evolution of elliptic flow as a function of energy seen in Fig. 10 is understood qualitatively rather well. At low energies, in-plane $\left(v_{2}>0\right)$, rotation-like, emission may arise due to low energy density in the overlap region and of long reaction times. The fast transition towards preferential emission out-of-plane $\left(v_{2}<0\right)$ is the outcome of more energetic collisions, leading to a larger energy density of the fireball. The increase of elliptic flow is a fingerprint of a stronger collective expansion, hindered by the passing spectators, which act as a shadow for the outgoing nucleons and fragments. The competition between the increasing speed of the expansion and of the decreasing passage time $t_{\text {pass }}$ of spectators leads to a maximum of (absolute value) elliptic flow in the SIS energy range. In this energy domain, the transiting spectators, with $t_{\text {pass }}$ varying between 40 and $10 \mathrm{fm} / c$, act as a clock for the collective expansion. In this regime, elliptic flow (historically called "squeeze-out", [122,123]) is a prominent observable for the extraction of the nuclear EoS [14]. Towards larger energies, elliptic 


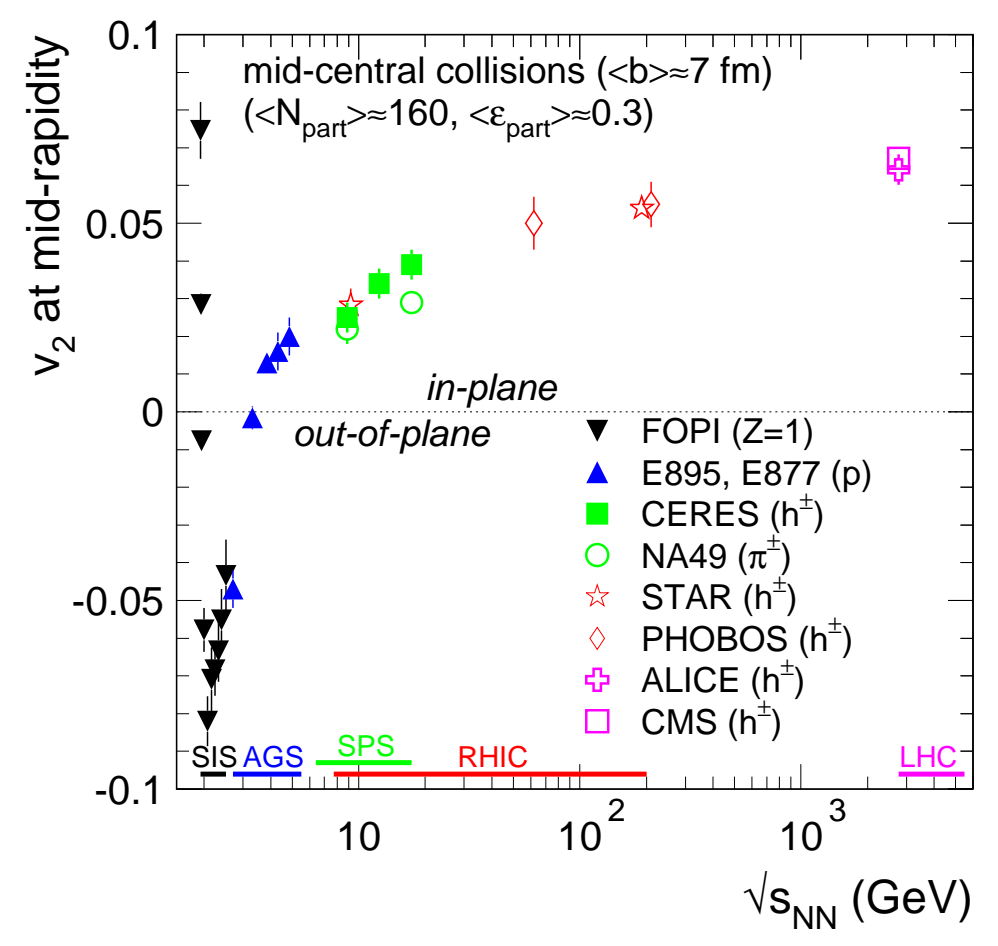

Figure 10: Collision energy dependence of the elliptic flow parameter $v_{2}$, measured at midrapidity in mid-central collisions.

flow exhibits another transition, to a preferential in-plane emission [124], a result of a unhindered collective expansion of the initially-anisotropic fireball. Elliptic flow is built mostly in the earlier stages of the collision, since it is determined by the initial pressure gradients, which it alters quickly as it develops. Consequently, at high energies, elliptic flow probes (albeit not exclusively) the deconfined state of quarks and gluons.

The elliptic flow measurement at the LHC [120] exhibits a magnitude about 30\% larger compared to the measurement at $\sqrt{s_{\mathrm{NN}}}=200 \mathrm{GeV}$. This increase is described by hydrodynamics and was anticipated on a purely phenomenological $\log \left(\sqrt{s_{\mathrm{NN}}}\right)$ behavior seen at lower energies [43]. The data show [120] that the $p_{\mathrm{T}}$ dependence of $v_{2}$ is identical at the LHC to that measured at RHIC, implying that the increase for the $p_{\mathrm{T}}$-integrated $v_{2}$ value is due exclusively to the increase of the average transverse momentum of the hadrons. The scaling of $v_{2}$ with the number of valence quarks observed at RHIC [125, 126] seems less well obeyed at the LHC [127].

A good description of spectra and elliptic flow measured at the LHC is achieved in hydrodynamical models, both at RHIC and at the LHC energies, see Ref. [69, 127] for the LHC case; an illustration for the VISHNU model [128] is shown in Fig. 11. Another component of flow, called directed flow, is quantified by the first Fourier coefficient, $v_{1}=\langle\cos (\phi)\rangle$. Directed flow is strong at lower energies, where it was observed observed for the first time [108, and is studied currently both at the LHC [129] and at RHIC [130] energies. Higher-order Fourier coefficients are also studied extensively [131,132], while further refinements based on multi-particle azimuthal correlations [133] address the question of collective phenomena in small systems. Flow-like features have been indeed identified recently in $\mathrm{p}-\mathrm{Pb}$ collisions at the LHC [134 137] and at RHIC [138]; some of these features were earlier predicted by hydrodynamical models [139], incorporating a collective expan- 

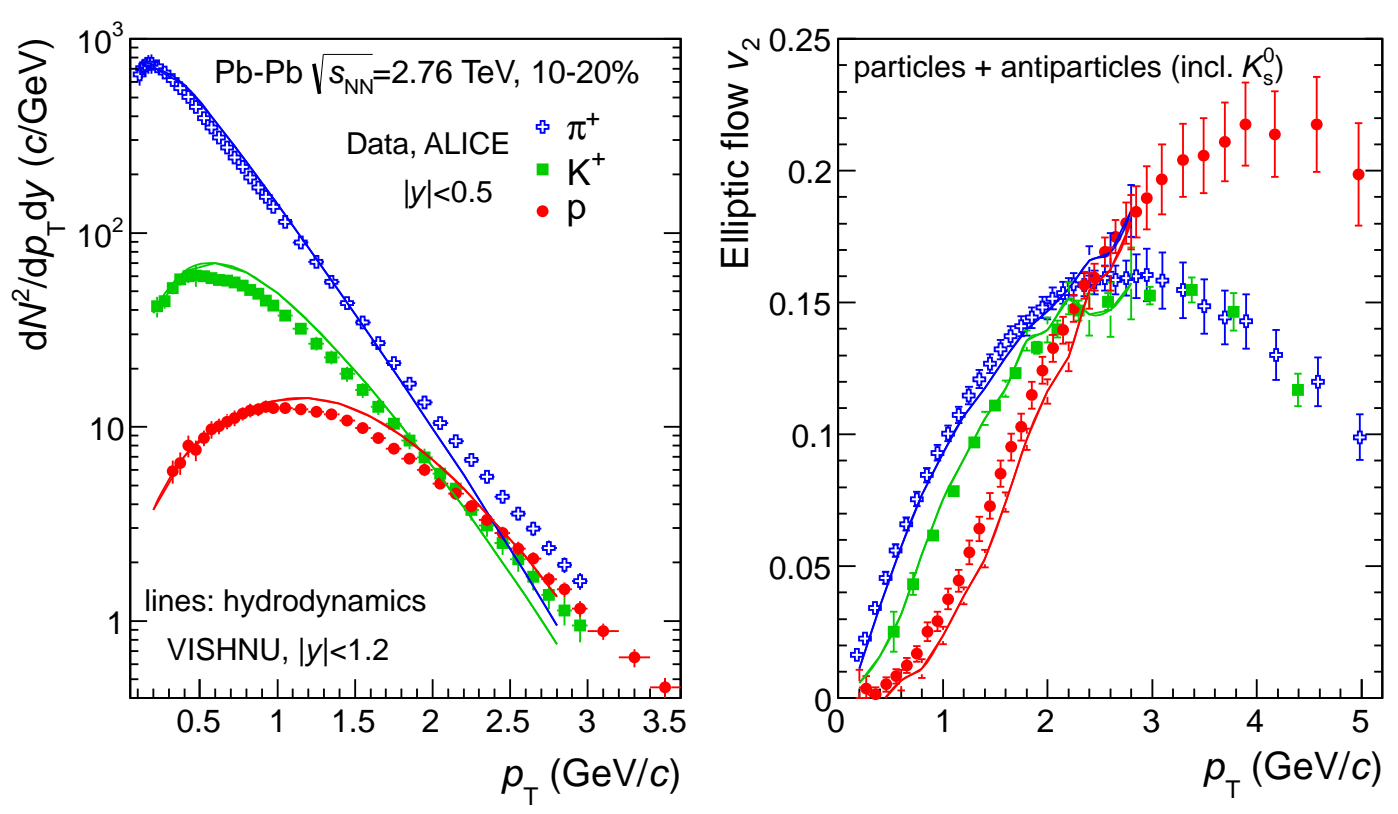

Figure 11: Transverse momentum spectra and the transverse momentum dependence of the elliptic flow parameter $v_{2}$ for pions, kaons and protons measured in $10-20 \%$ central $\mathrm{Pb}-$ $\mathrm{Pb}$ collisions at $\sqrt{s_{\mathrm{NN}}}=2.76 \mathrm{TeV}$ at the LHC [69, 127], in comparison to hydrodynamical model calculations [128].

sion akin to that in $\mathrm{Pb}-\mathrm{Pb}$ collisions. This is currently a subject of intense theoretical effort [140 145], that promises to bring further insights on flow effects in small volumes (but characterized by very large energy density).

Another set of experimental observables from the kinetic freeze-out stage are obtained from Bose-Einstein correlations of identical particles (called also femtoscopy, or Hanbury Brown and Twiss (HBT) interferometry) [146]. This enables the measurement of the spatial extension of a region of the fireball, the region of homegeneity, and the lifetime of the expanding fireball until the kinetic freeze-out. These measurements, performed over twenty years of heavy-ion collision studies, have recently been extended with data at the LHC [147] and at RHIC [148].

The energy dependence of the volume extracted at chemical freeze-out $V_{\text {chem }}$ and at kinetic freeze-out $V_{k i n}$ in central collisions is shown in Fig. 12, $V_{\text {chem }}$ corresponds to one unit of rapidity and is calculated from the $\mathrm{d} N_{c h} / \mathrm{d} y$ data (Fig. 2) and the densities calculated in the thermal model for parametrized $T$ and $\mu_{B}$ values. $V_{k i n}$ is obtained from femtoscopy radii and corresponds to the pion pair transverse momentum $k_{\mathrm{T}} \simeq 0.22$ $\mathrm{GeV} / c$ (data from Ref. [148], see references therein). Note that the kinetic freeze-out volume extracted from femtoscopy is not the entire volume of the fireball, but that of the region of homogeneity [146].

The energy dependence of the ("decoupling") time at kinetic freeze-out $\tau$, obtained from fits of $R_{\text {long }}\left(k_{\mathrm{T}}\right)$ assuming a one-dimensional (longitudinal) hydrodynamics expansion (the Bjorken model), is shown in Fig. 13. Hydrodynamical models [149,150], are successful in predicting the femtoscopy observables (see Ref. [147]).

Elliptic flow, radial flow and femtoscopy observables are used to extract, via comparisons to hydrodynamic calculations, the ratio of the shear viscosity to entropy density, $\eta / s[151])$. The remarkable description of flow and femtoscopy in hydrodynamic models 


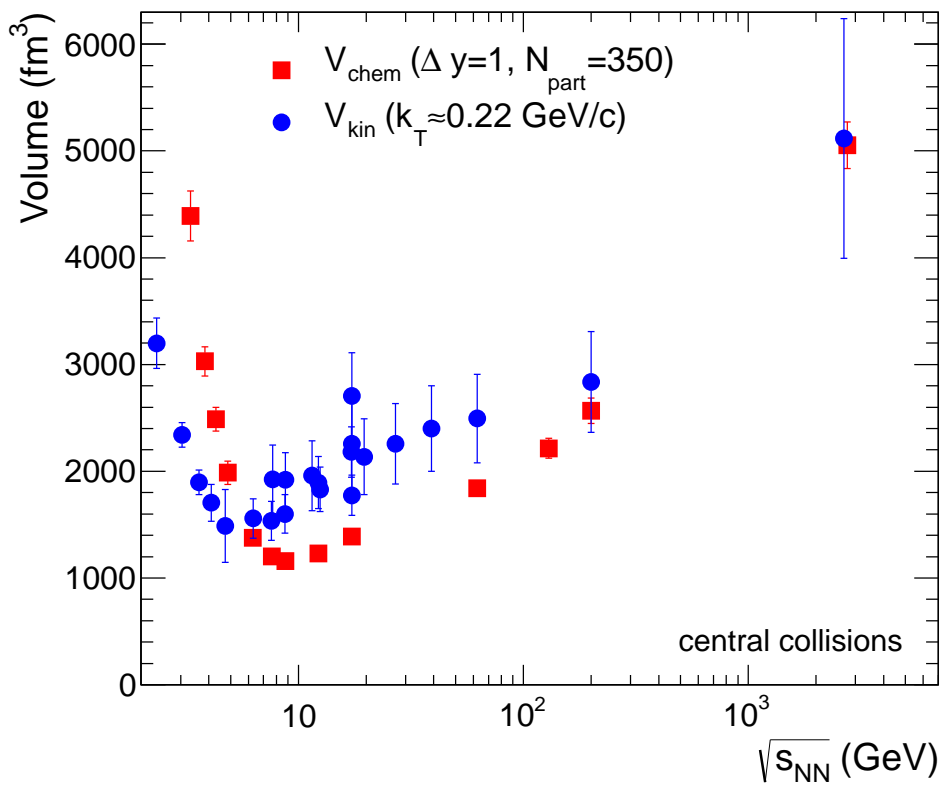

Figure 12: Collision energy dependence of the fireball volume extracted at chemical and at kinetic freeze-out in central collisions. The data for the kinetic freeze-out volume is from Ref. [148].

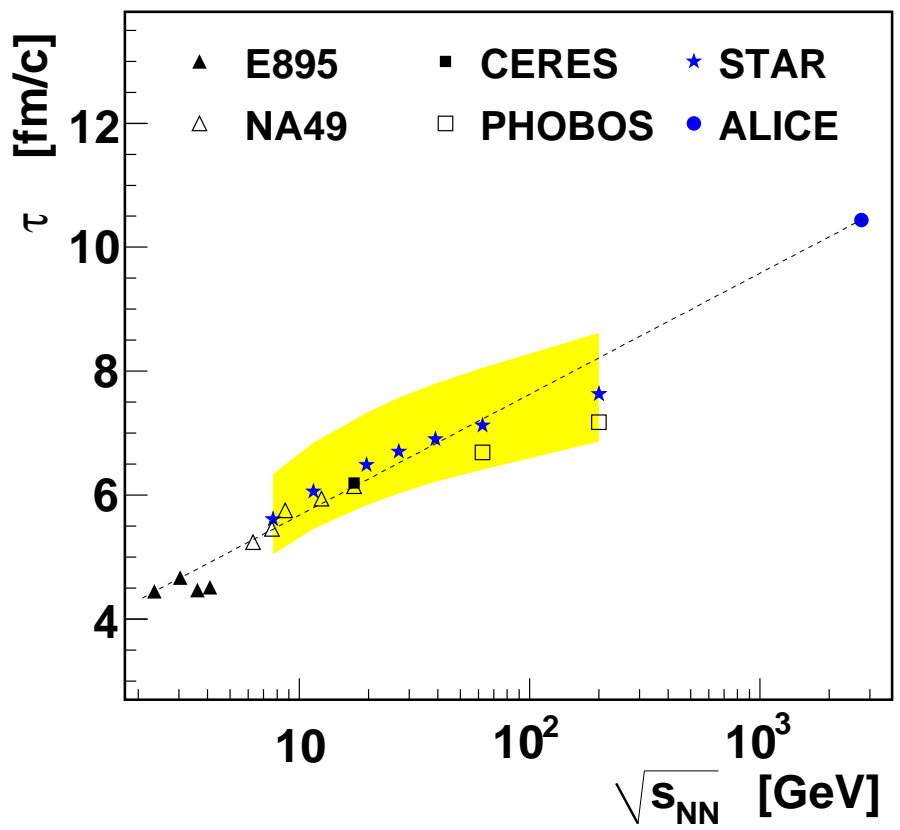

Figure 13: Collision energy dependence of the duration until kinetic freeze-out, $\tau$, for central collisions (plot from Ref. [148], see references therein). The yellow band denotes the uncertainty in $\tau$ due to the uncertainty in the freeze-out temperature [148].

observed at RHIC is further confirmed and extended with the measurements at the LHC. The quantitative determination of $\eta / s$ is dependent on fine details of the initial energy density distribution [152], which can be calculated, based on constraints on average values from data, according to either the Glauber model or in the Color Glass Condensate framework; recent work [153] aims at quantitative constraints for the initial conditions. 
Deconfined matter is characterized by low values of $\eta / s$ [152, 154, 155] and it remains a challenge to establish the possible existence of a lower bound [156]; for values extracted at much lower collision energies see Ref. [157, 158; an estimate for nuclear matter in mildly-excited nuclei is given in Ref. [159]. Thermalization at a very early stage of the collision, at or below $1 \mathrm{fm} / c$, as employed in hydrodynamic calculations, remains a challenge to theory [160], but recent developments [161] bring further hope for understanding the issue.

Can we identify an onset of deconfinement based on the (bulk) hadronic observables discussed above? The change, in the range $\sqrt{s_{\mathrm{NN}}} \simeq 5-10 \mathrm{GeV}$, of fireball properties (see Fig. 9. 10) is a possible fingerprint, but further experimental and theoretical support is needed to conclude.

Probing the deconfined matter in a more direct way is done with special observables of the early stage. Proposed early on as thermometers of the deconfined stage, thermal photons and low-mass dileptons [162] (see Ref. [163, 164] for recent reviews) are such observables. Low-mass dileptons are also probes of the chiral symmetry restoration in hot QCD matter [165]. Measurements of thermal photons at RHIC have shown [166] (see also Ref. [167]) that the temperature averaged over the lifetime of the fireball is larger than the chemical freeze-out $T$. A somewhat larger $T$ value is extracted from preliminary data of photon production at the LHC [168]. The observation of elliptic flow of thermal photons at RHIC [169], observed also at the LHC [170], made more complex the connection between the thermal photon measurements and the hottest stage of the system [171,172]. This is a challenging and fascinating field of investigation, both experimentally and theoretically, from which crucial insights are expected to arise in the coming years.

Another category of QGP probes is the so-called hard probes, namely processes char-

acterized by an energy scale (quantified by the transverse mass $m_{\mathrm{T}}=\sqrt{m_{0}^{2}+p_{\mathrm{T}}^{2}}$, where $m_{0}$ is the rest mass of the hadron) above several $\mathrm{GeV}$ (well above the temperature of the medium). Examples of such observables are hadrons at high $p_{\mathrm{T}}$ (or jets) [173] and hadrons containing heavy (charm or bottom) quarks [174]; they are produced at early times in the collision, $t=1 / m_{\mathrm{T}}$. Quarkonium formation [175] is another prominent observable for deconfined matter studies; sections 5 and 6 present selected results on these measurements and their interpretation in theoretical models.

\section{$5 \quad$ Parton energy loss}

Proposed by Bjorken in 1982 [176], "jet quenching", the extinction of jets (due to the energy loss of the parent parton) in QGP was for the first time observed at RHIC [177, 178] and is a subject of intense study at the LHC [179-183]. The usual method to quantify jet quenching is via the nuclear modification factor, defined as:

$$
R_{\mathrm{AA}}=\frac{\mathrm{d}^{2} N_{\mathrm{A} A} / \mathrm{d} y \mathrm{~d} p_{\mathrm{T}}}{N_{\text {coll }} \cdot \mathrm{d}^{2} N_{\mathrm{pp}} / \mathrm{d} y \mathrm{~d} p_{\mathrm{T}}},
$$

where $\mathrm{d}^{2} N / \mathrm{d} y \mathrm{~d} p_{\mathrm{T}}$ denotes the yield of a given observable measured in nucleus-nucleus (AA) or proton-proton (pp) collisions and $N_{\text {coll }}$ is the average number of nucleon-nucleon collisions over the given centrality interval of AA collisions (see Fig. 1).

A change of physics in AA collisions (which in specialized terms is called a "medium effect") is seen as a departure of $R_{\mathrm{AA}}$ from unity. Modifications of parton distributions in nuclei compared to free nucleons, denoted as "shadowing" or "saturation", need to 
be considered carefully, in particular at LHC energies. Measurements in $\mathrm{p}-\mathrm{Pb}$ collisions address this issue [184].

We note that the binary collision scaling assumed in the construction of $R_{\mathrm{AA}}$ applies only to hard processes. It is known experimentally that bulk particle production (comprising essentially pions, protons and kaons at low-momentum, $p_{\mathrm{T}} \lesssim 3-4 \mathrm{GeV} / c$ ) in AA collisions scales (in first order) with $N_{\text {part }}$ [4] (see Fig. 33).

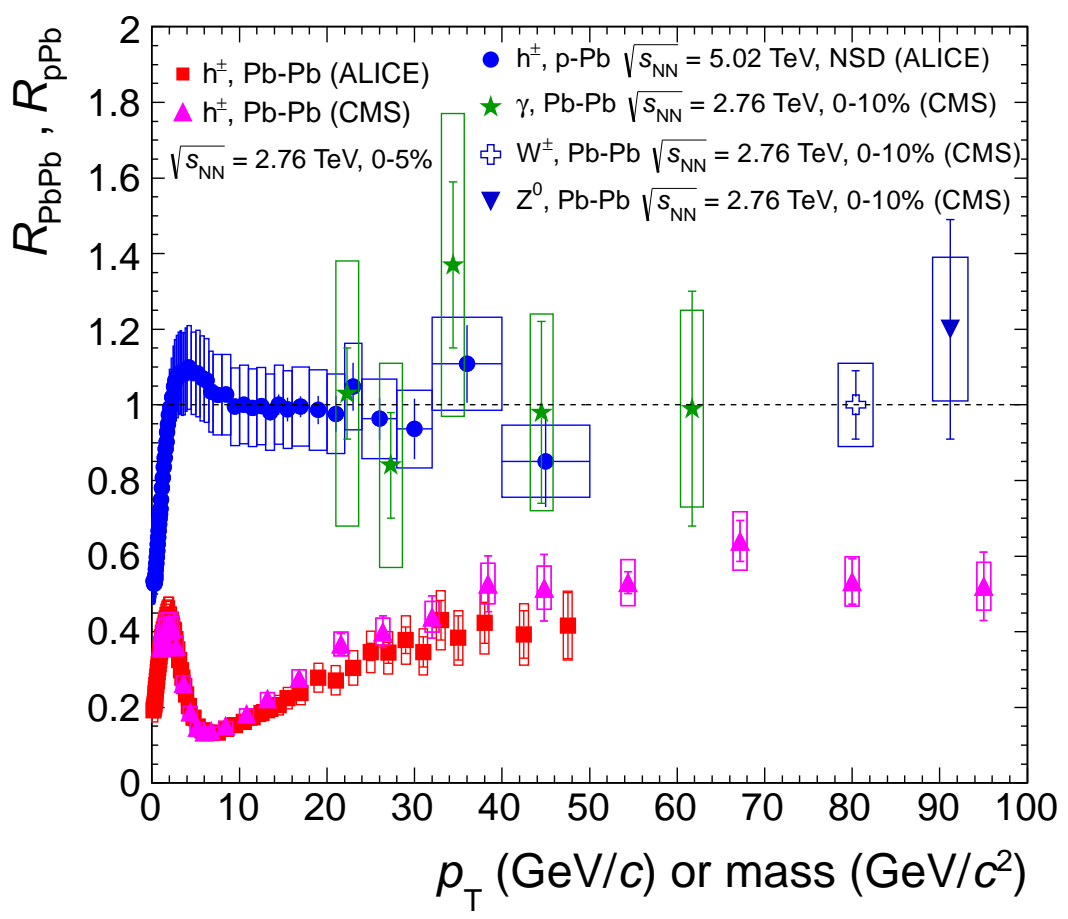

Figure 14: Transverse momentum dependence of the nuclear modification factor $R_{\mathrm{pPb}}$ of charged particles $\left(\mathrm{h}^{ \pm}\right)$measured in minimum-bias (NSD) $\mathrm{p}-\mathrm{Pb}$ collisions at $\sqrt{s_{\mathrm{NN}}}=$ $5.02 \mathrm{TeV}$ [185] in comparison to data on the nuclear modification factor $R_{\mathrm{PbPb}}$ in central $\mathrm{Pb}-\mathrm{Pb}$ collisions at $\sqrt{s_{\mathrm{NN}}}=2.76 \mathrm{TeV}$. The $\mathrm{Pb}-\mathrm{Pb}$ data are for charged particle [180,181], direct photon [186], $\mathrm{Z}^{0}$ [187], and $\mathrm{W}^{ \pm}$[188] production. All data are for midrapidity (plot from Ref. [185]).

The first measurement of $R_{\mathrm{AA}}$ for inclusive charged-hadron production at the LHC [179] showed that the suppression is larger than previously measured at RHIC, reaching a factor of about 7 . The suppression is reduced towards larger $p_{\mathrm{T}}$ values, see Fig. 14, but remains substantial even at 50-100 GeV/c [180, 181]. Recent data for $\mathrm{p}-\mathrm{Pb}$ collisions at the LHC [185] demonstrate that the suppression of hadron production at high $p_{\mathrm{T}}$ in $\mathrm{Pb}-\mathrm{Pb}$ collisions, has no contribution from initial state effects. The ALICE $\mathrm{p}-\mathrm{Pb}$ data show no sign of nuclear matter modification of hadron production at high $p_{\mathrm{T}}$ and are therefore fully consistent with the observation of binary collision scaling in $\mathrm{Pb}-\mathrm{Pb}$ of observables which are not affected by hot QCD matter (direct photons [186] and vector bosons [187, 188]).

The measurements, in conjunction with theoretical models, clearly demonstrate that partons lose energy in the deconfined hot and dense matter created in collisions at RHIC and LHC. The basic features seen in the data are reproduced by models, see Fig. 15] for the LHC energy. The ultimate goal of such studies is the extraction of transport coefficients; presently, large uncertainties are originating from the description of jet quenching in 


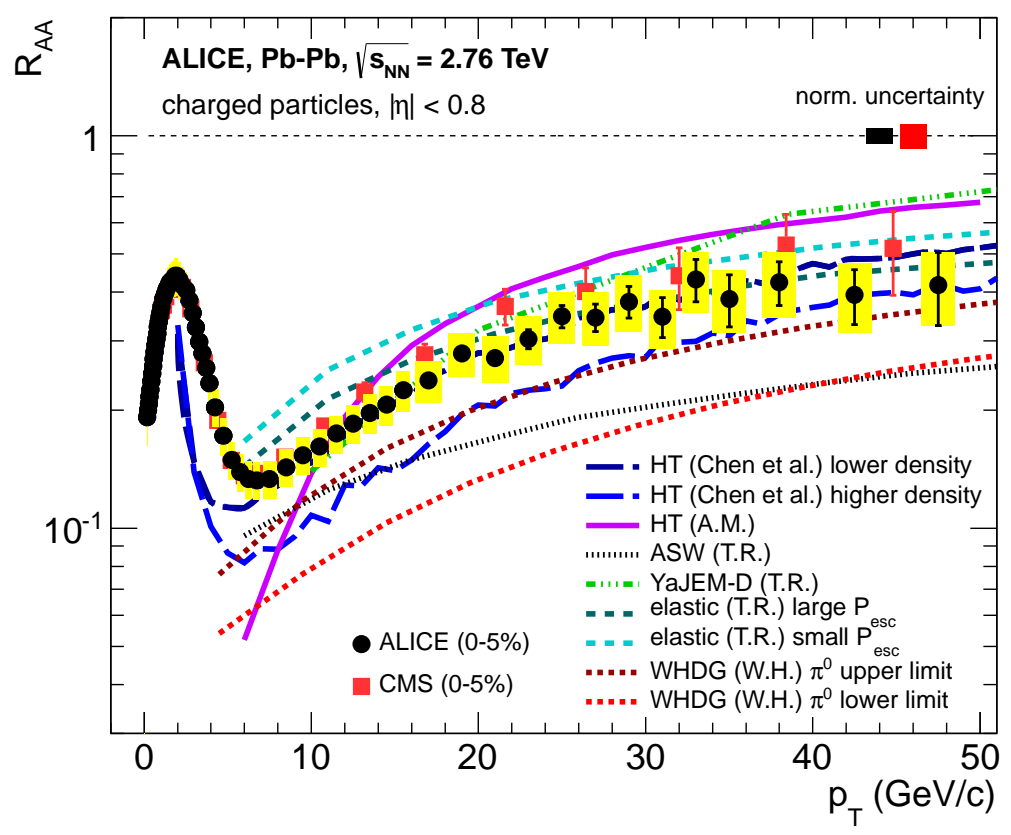

Figure 15: The nuclear modification factor $R_{\mathrm{AA}}$ as a function of transverse momentum for inclusive charged hadron production in $\mathrm{Pb}-\mathrm{Pb}$ collisions at the LHC (Fig. from Ref. [181], see references therein for the theoretical curves).

theoretical models, which remains a challenging task [189]. The spread of the theoretical curves in Fig. 15 illustrates this challenge. Recent progress on the theoretical front and the availability of data at the LHC allows already a quantitative extraction of the jet quenching parameter $\hat{q}$ (which is the average squared transverse momentum acquired by the parton per unit path length) in deconfined QCD matter, a quantity which has recently been calculated in lattice QCD [190]. Values of $\hat{q}$ of several $\mathrm{GeV}^{2} / \mathrm{fm}$ are extracted in the systematic study of Ref. [191].

The nuclear modification factor for production of hadrons carrying charm [192] or bottom [193] quarks is shown in Fig. 16] as a function of centrality in $\mathrm{Pb}-\mathrm{Pb}$ collisions at the LHC. The theoretical expectation is that heavy quarks (charm and bottom) lose less energy (by gluon radiation) compared to lighter (up, down, strange) ones [194]. This expectation appears to be exhibited by the data, see Fig. 16, although a definite conclusion needs further support from experiment.

The energy loss suffered by energetic heavy quarks in QGP is indicative of their "strong coupling" with the medium, dominated by light quarks and gluons. The measurements at the LHC [195] consolidate and extend the observation at RHIC of heavy quark energy loss and flow [196. Theoretical models of quark energy loss in deconfined matter describe the data well, although a large spread exists between various model predictions (see Ref. [195] and references therein). New data at RHIC with reconstructed $D^{0}$ mesons [197] indicate similar mechanisms of energy loss compared to the LHC case. Measurements of elliptic flow of heavy quarks at the LHC [198] and at RHIC [196,199] impose additional constraints to the theoretical models [200].

Experimental studies at the LHC with reconstructed jets [183, 201, 202 indicate a redistribution of the energy inside the jet cone [201]. Measurements of jets with bottom quarks indicate that parton energy loss at higher parton energies $(80-250 \mathrm{GeV})$ is not 


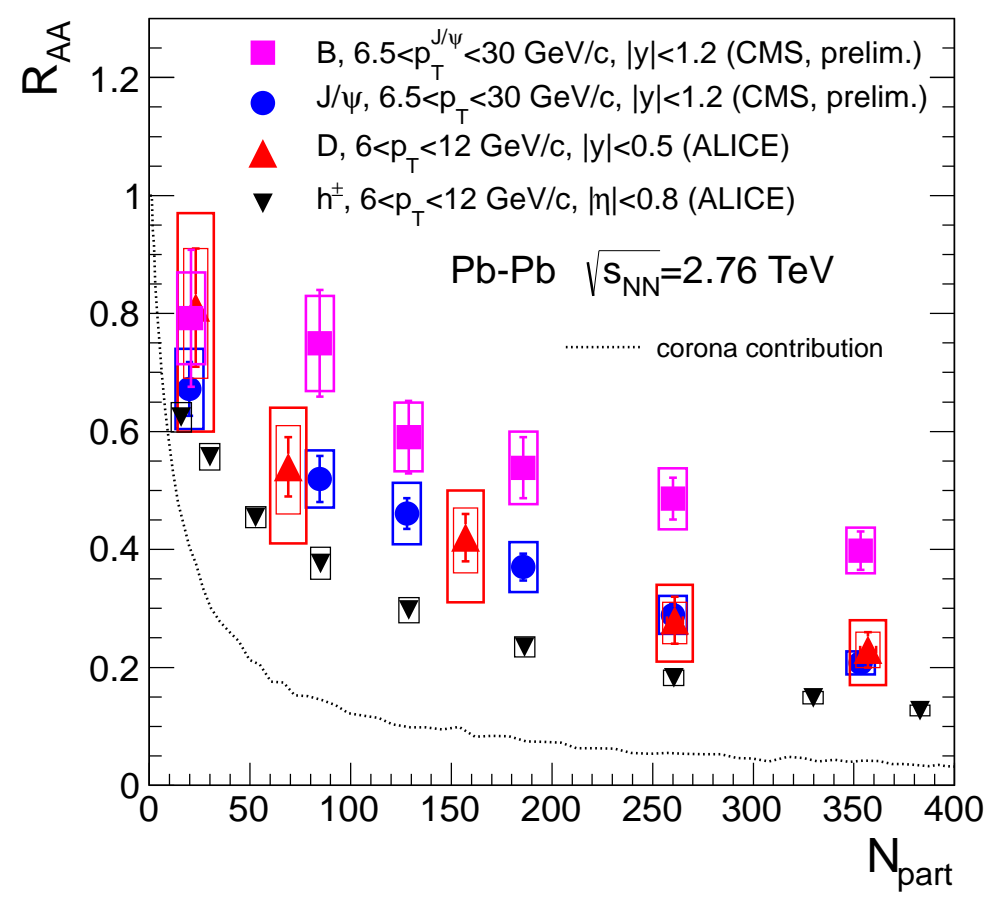

Figure 16: Centrality dependence of the nuclear modification factor $R_{\mathrm{AA}}$ for charged hadrons at high $p_{\mathrm{T}}$ in comparison to that of charmed mesons $(D)$, charmonium $(\mathrm{J} / \psi)$ and beauty hadrons $(B)$. The dotted line is an estimate of the lowest $R_{\mathrm{AA}}$ value, originating from the production in the corona of colliding nuclei.

dependent on parton mass (flavor) [203].

\section{Quarkonium}

Among the various suggested probes of deconfinement, charmonium $(c \bar{c})$ states plays a distinctive role. The $\mathrm{J} / \psi$ meson is the first hadron for which a clear mechanism of suppression (melting) in QGP was proposed early on, based on the color analogue of Debye screening [175]. Further refinements, including the whole quarkonia species, $c \bar{c}$ and $b \bar{b}$, led to the picture of "sequential suppression" [204 206], a hierarchy of quarkonium dissociation dependent on the binding energy (size) of the quarkonium state, which could give information on the temperature of the medium (given that the Debye length in deconfined matter has a pronunced temperature dependence [205]). It was pointed out early-on that the Debye screening phenomenon is a low- $p_{\mathrm{T}}$ effect [204]. A review of data and its interpretation in the screening scenario is available in Ref. [207]. Lattice QCD calculations can give information on the screening, see earlier arguments [208 210] and more recent work [211 216] (see a review in Ref. [217]).

Novel quarkonium production mechanisms were proposed. In the statistical hadronization model [218], the charm quarks produced in initial hard collisions thermalize in QGP and are "distributed" into hadrons at chemical freeze-out. All charmonium states are assumed to be not formed at all in the deconfined state but are produced, together with all other hadrons, at chemical freeze-out [218,219] (see Ref. [75,220] for recent predictions of this model). Kinetic (re)combination of charm and anti-charm quarks in QGP [221] is an alternative quarkonium production mechanism. In this model (see Ref. [222 225] 
for recent results), continuous dissociation and (re)generation of charmonium takes place over the entire lifetime of the deconfined stage.
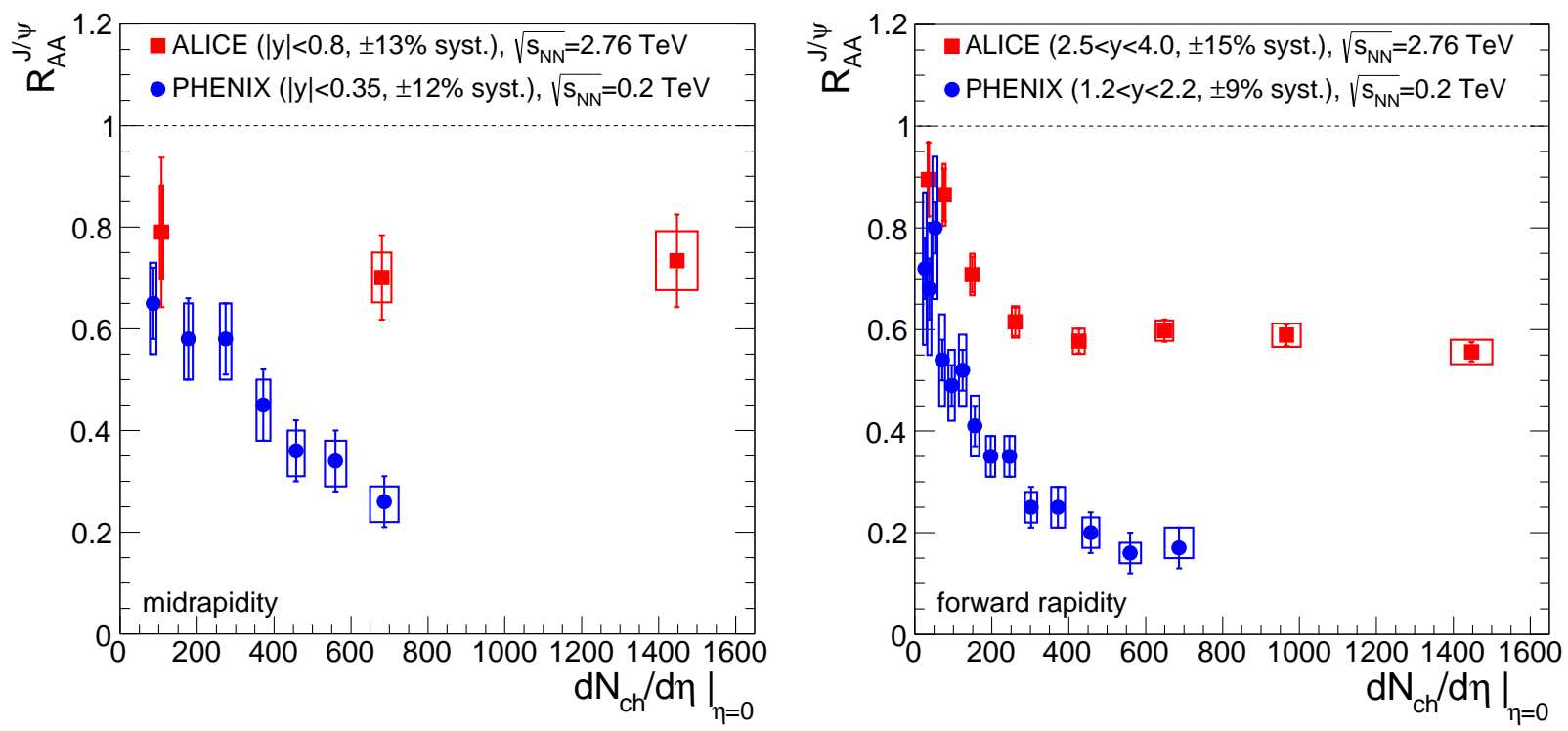

Figure 17: The dependence of the nuclear modification factor $R_{\mathrm{AA}}$ for inclusive $\mathrm{J} / \psi$ production on the multiplicity density (at $\eta=0$ ) for midrapidity (left panel) and at forward rapidity (right panel). The data are integrated over $p_{\mathrm{T}}$ and are from the PHENIX collaboration [226] at RHIC and ALICE collaboration [227] at the LHC. Note the overall uncertainties of the data quoted in the legend.

The measurement of $\mathrm{J} / \psi$ production in $\mathrm{Pb}-\mathrm{Pb}$ collisions at the $\mathrm{LHC}$ was expected to provide a definitive answer on the question of (re)generation. The data measured at high $p_{\mathrm{T}}$ [228] show a pronounced suppression of $\mathrm{J} / \psi$ in $\mathrm{Pb}-\mathrm{Pb}$ compared to pp collisions and of the same magnitude as that of open-charm hadrons, see Fig. 16. This may indicate that the high- $p_{\mathrm{T}}$ charm quarks that form either $D$ or $\mathrm{J} / \psi$ mesons had the same dynamics, possibly a thermalization in QGP and a late hadronization.

The first LHC measurement of the overall (inclusive in $p_{\mathrm{T}}$ ) production [230], showed for foward rapidities $R_{\mathrm{AA}}$ values significantly larger than at RHIC energies. More recent data [227] confirmed this, see Fig. 17. The data are well described by both the statistical hadronization model [75] and by transport models [222, 223], as demonstrated in Fig. 18. Based on these observations, the $\mathrm{J} / \psi$ production can be considered a probe of deconfinement as initially proposed [175, but may not be a "thermometer" of the medium. Within the statistical model, the charmonium states become probes of the phase boundary between the deconfined and the hadron phases. This extends the family of quarks employed for the determination of the hadronization temperature (via the conjectured connection to the chemical freeze-out temperature extracted from fits of statistical model calculations to hadron abundances discussed above).

In transport models, about $60-70 \%$ of the $\mathrm{J} / \psi$ yield in $\mathrm{Pb}-\mathrm{Pb}$ collisions originates from (re)combination of $c$ and $\bar{c}$ quarks, the rest being primordial $\mathrm{J} / \psi$ mesons that have survived in the deconfined medium. These models show [223, 225] that, as expected, (re)generation is predominantly a low- $p_{\mathrm{T}}$ phenomenon, as illustrated by the measured $p_{\mathrm{T}}$ dependence of $R_{\mathrm{AA}}$, shown in Fig. 19. The measurement of $\mathrm{J} / \psi$ elliptic flow at the LHC [231], albeit to date of limited statistical significance, brings another argument in favor of charm quark thermalization. The $\mathrm{J} / \psi$ data at RHIC are compatible with a null 


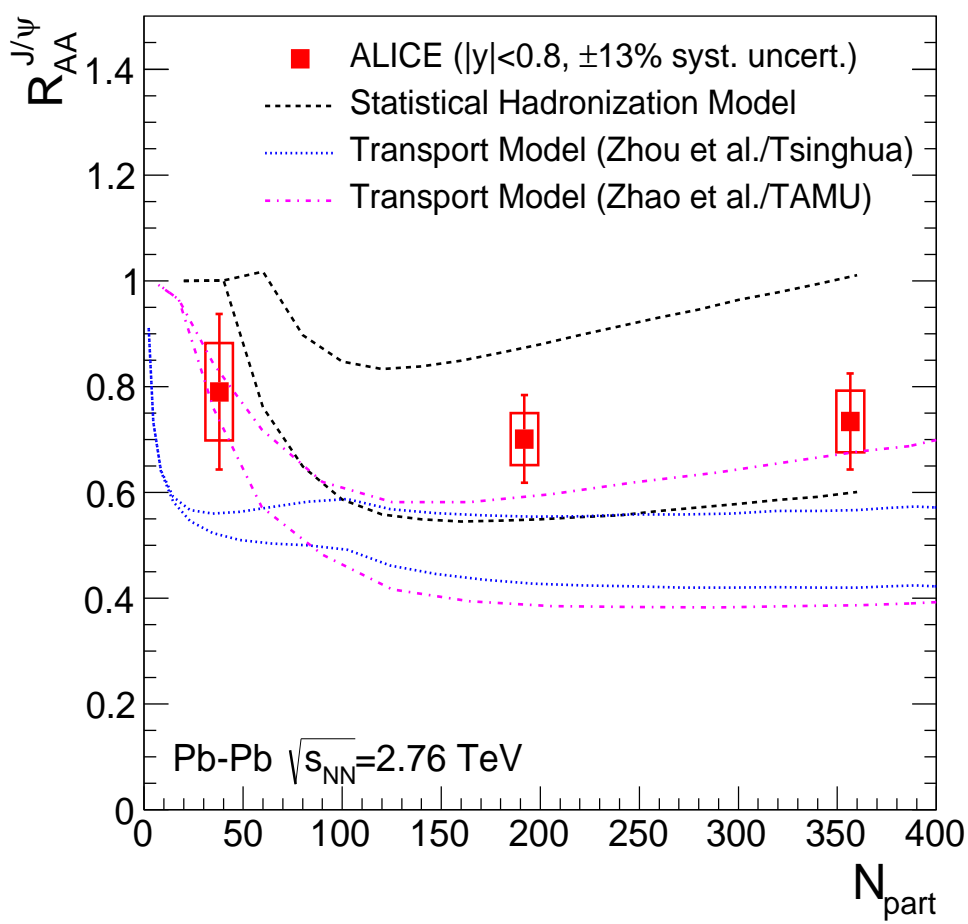

Figure 18: Centrality dependence of the nuclear modification factor for inclusive $\mathrm{J} / \psi$ production at the LHC. The data at midrapidity [227] are compared to model calculations: the statistical hadronization model [75] and transport models of the TAMU [223,229] and Tsinghua [222,225] groups. The bands are incorporating (part of) the uncertainty in the charm production cross section.

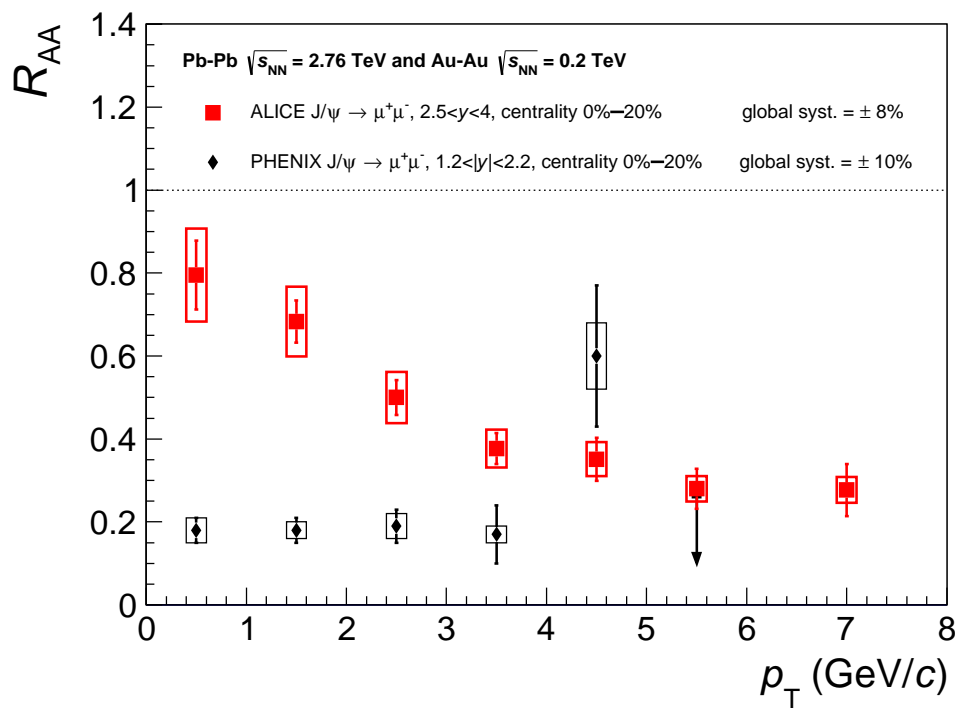

Figure 19: Transverse momentum dependence of the nuclear modification factor for $\mathrm{J} / \psi$ production measured at RHIC [226] and at the LHC [227] (plot from Ref. [227]).

flow signal 232. A $v_{2}$ signal was measured for $\mathrm{J} / \psi$ at the SPS [233] and was interpreted as a path-length dependence of the screening.

The quarkonium data at the LHC demonstrate that (re)generation in deconfined matter during the QGP lifetime or at the chemical freeze-out are the only possible mechanisms 
of production. Discriminating the two pictures will help providing an answer to fundamental questions related to the fate of (quarkonium) hadrons in a hot medium [209, 234, 235. Data at the top LHC energy, including measurements on $\psi(2 S)$ production, will help clarifying such questions. Recent measurements of charmonium production in p-A collisions at the LHC [236, 237] could help constraining models. Interesting aspects are revealed by the measurement of $\psi(2 S)$ production in $\mathrm{d}-\mathrm{Au}$ collisions at RHIC [238] and in $\mathrm{p}-\mathrm{Pb}$ at the LHC [239], indicating possible final-state effects.

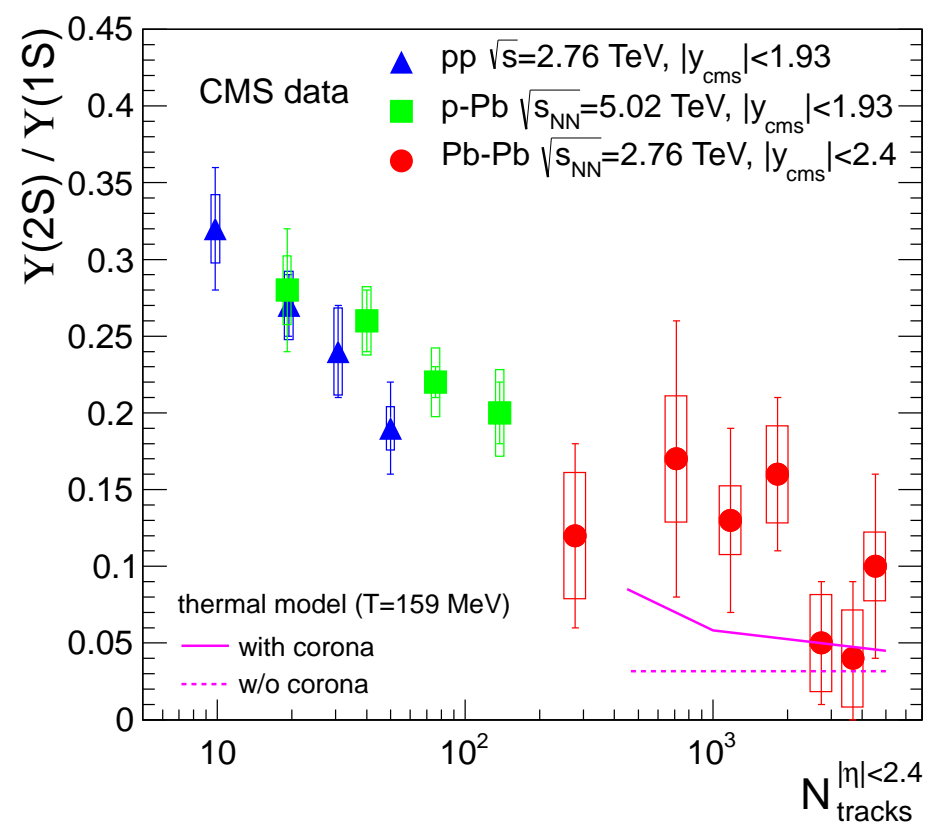

Figure 20: Track multiplicity dependence of the ratio of yields if $\Upsilon(2 S)$ and $\Upsilon(1 S)$ bottomonium states, measured at the LHC in $\mathrm{pp}, \mathrm{p}-\mathrm{Pb}$ and $\mathrm{Pb}-\mathrm{Pb}$ collisions [240]. The lines are thermal model predictions for central $\mathrm{Pb}-\mathrm{Pb}$ collisions; the full line includes an estimate of the contribution of the production in the corona of the colliding nuclei.

Recent measurements of production of bottomonium $(b \bar{b})$ states at the LHC [240]242] and at RHIC [243] add an important new aspect to the quarkonium dissociation story. The nuclear modification factor for the $\Upsilon$ states exhibits at the LHC a sequential suppression pattern 241. Transport model predictions 224] describe the data (albeit not in detail at the LHC [242]) while the Debye screening picture implemented in a hydrodynamical approach [244] is less successful (see Ref. [242]). The production ratio $\Upsilon(2 S) / \Upsilon(1 S)$, shown in Fig. 20, is very different in $\mathrm{Pb}-\mathrm{Pb}$ compared to pp collisions [245]; the data are compatible, for central $\mathrm{Pb}-\mathrm{Pb}$ collisions, to the value predicted by the statistical hadronization model for $T=159 \mathrm{MeV}$. This provides a tantalizing possibility of adding the bottom flavor towards constraining even further the QCD phase boundary with nucleusnucleus data at high energies.

\section{References}

[1] G. Chapline, M. Johnson, E. Teller, et al., Phys. Rev. D 8, 4302 (1973)

[2] J. C. Collins, M. Perry, Phys. Rev. Lett. 34, 1353 (1975) 
[3] N. Cabibbo, G. Parisi, Phys. Lett. B 59, 67 (1975)

[4] G. Chapline, M. Nauenberg, Phys. Rev. D 16, 450 (1977)

[5] E. V. Shuryak, Phys. Lett. B 78, 150 (1978)

[6] D. Boyanovsky, H. de Vega, D. Schwarz, Ann. Rev. Nucl. Part. Sci. 56, 441 (2006), hep-ph/0602002

[7] M. G. Alford, K. Schwenzer (2013), 1310.3524

[8] P. Braun-Munzinger, J. Wambach, Rev. Mod. Phys. (2008), 0801.4256

[9] P. Braun-Munzinger, J. Stachel, Nature 448, 302 (2007)

[10] B. V. Jacak, B. Müller, Science 337, 310 (2012)

[11] B. Müller, Lecture at the Nobel Symposium on LHC Results (2013), 1309.7616

[12] H. Satz, Int. J. Mod. Phys. A 28, 1330043 (2013), 1310.1209

[13] J. Schukraft, Lecture at the Nobel Symposium on LHC Results (2013), 1311.1429

[14] P. Danielewicz, R. Lacey, W. G. Lynch, Science 298, 1592 (2002), nucl-th/0208016

[15] T. Klahn, D. Blaschke, R. Lastowiecki, Acta Phys. Polon. Supp. B 5, 757 (2012), 1111.6889

[16] F. Karsch, Lect. Notes Phys. 583, 209 (2002), hep-lat/0106019

[17] Y. Aoki, G. Endrodi, Z. Fodor, et al., Nature 443, 675 (2006), hep-lat/0611014

[18] Y. Aoki, S. Borsanyi, S. Dürr, et al., JHEP 0906, 088 (2009), 0903.4155

[19] A. Bazavov, T. Bhattacharya, M. Cheng, et al., Phys. Rev. D 85, 054503 (2012), 1111.1710

[20] M. Aggarwal, et al. (STAR Collaboration) (2010), 1007.2613

[21] O. Philipsen, Acta Phys. Polon. Supp. 5, 825 (2012), 1111.5370

[22] R. V. Gavai (2014), 1404.6615

[23] F. Karsch, PoS CPOD2013, 046 (2013), 1307.3978

[24] J. Gosset, H. Gutbrod, W. Meyer, et al., Phys. Rev. C 16, 629 (1977)

[25] J. Aichelin, Phys. Rept. 202, 233 (1991)

[26] H. Petersen, M. Bleicher, S. A. Bass, et al. (2008), 0805.0567

[27] O. Buss, T. Gaitanos, K. Gallmeister, et al., Phys. Rept. 512, 1 (2012), 1106.1344

[28] P. Huovinen, P. Ruuskanen, Ann. Rev. Nucl. Part. Sci. 56, 163 (2006), nucl-th/0605008 
[29] C. Gale, S. Jeon, B. Schenke, Int. J. Mod. Phys. A 28, 1340011 (2013), 1301.5893

[30] U. W. Heinz, J. Phys. Conf. Ser. 455, 012044 (2013), 1304.3634

[31] H. Petersen, J. Steinheimer, G. Burau, et al., Phys. Rev. C 78, 044901 (2008), 0806.1695

[32] B. Abelev, et al. (ALICE Collaboration), Phys. Rev. C 88, 044909 (2013), 1301.4361

[33] M. L. Miller, K. Reygers, S. J. Sanders, et al., Ann. Rev. Nucl. Part. Sci. 57, 205 (2007), nucl-ex/0701025

[34] B. Abelev, et al. (ALICE Collaboration), Eur. Phys. J. C 73, 2456 (2013), 1208.4968

[35] I. Arsene, et al. (BRAHMS Collaboration), Nucl. Phys. A 757, 1 (2005), nucl-ex/0410020

[36] K. Adcox, et al. (PHENIX Collaboration), Nucl. Phys. A 757, 184 (2005), nucl-ex/0410003

[37] B. Back, et al., Nucl. Phys. A 757, 28 (2005), nucl-ex/0410022

[38] J. Adams, et al. (STAR Collaboration), Nucl. Phys. A 757, 102 (2005), nucl-ex/0501009

[39] B. Muller, J. L. Nagle, Ann. Rev. Nucl. Part. Sci. 56, 93 (2006), nucl-th/0602029

[40] B. Muller, J. Schukraft, B. Wyslouch, Ann. Rev. Nucl. Part. Sci. 62, 361 (2012), 1202.3233

[41] B. Abelev, et al. (ALICE Collaboration), Phys. Rev. Lett. 105, 252301 (2010), 1011.3916

[42] E. Shuryak, Physics 3, 105 (2010), URL http://link.aps.org/doi/10.1103/Physics.3.105

[43] A. Andronic, P. Braun-Munzinger, Lect. Notes Phys. 652, 35 (2004), hep-ph/0402291

[44] K. Aamodt, et al. (ALICE Collaboration), Phys. Rev. Lett. 106, 032301 (2011), 1012.1657

[45] F. Gelis, E. Iancu, J. Jalilian-Marian, et al., Ann. Rev. Nucl. Part. Sci. 60, 463 (2010), 1002.0333

[46] F. Gelis, Int. J. Mod. Phys. A 28, 1330001 (2013), 1211.3327

[47] S. Chatrchyan, et al. (CMS Collaboration), Phys. Rev. Lett. 109, 152303 (2012), 1205.2488

[48] J. Bjorken, Phys. Rev. D 27, 140 (1983)

[49] J. Klay, et al. (E895 Collaboration), Phys. Rev. Lett. 88, 102301 (2002), nucl-ex/0111006 
[50] J. Klay, et al. (E895 Collaboration), Phys. Rev. C 68, 054905 (2003), nucl-ex/0306033

[51] C. Pinkenburg, et al. (E895 Collaboration), Nucl. Phys. A 698, 495 (2002), nucl-ex/0104025

[52] L. Ahle, et al. (E866 Collaboration, E917 Collaboration), Phys. Lett. B 476, 1 (2000), nucl-ex/9910008

[53] L. Ahle, et al. (E866 Collaboration, E917 Collaboration), Phys. Lett. B 490, 53 (2000), nucl-ex/0008010

[54] S. Ahmad, B. Bonner, S. Efremov, et al., Nucl. Phys. A 636, 507 (1998), nucl-ex/9803006

[55] S. Afanasiev, et al. (NA49 Collaboration), Phys. Rev. C 66, 054902 (2002), nucl-ex/0205002

[56] C. Alt, et al. (NA49 Collaboration), Phys. Rev. C 77, 024903 (2008), 0710.0118

[57] C. Alt, et al. (NA49 Collaboration), Phys. Rev. C 73, 044910 (2006), nucl-ex/0512033

[58] C. Alt, et al. (NA49 Collaboration), Phys. Rev. C 78, 034918 (2008), 0804.3770

[59] I. Bearden, et al. (NA44 Collaboration), Phys. Rev. C 66, 044907 (2002), nucl-ex/0202019

[60] F. Antinori, et al. (NA57 Collaboration), Phys. Lett. B 595, 68 (2004), nucl-ex/0403022

[61] B. Abelev, et al. (STAR Collaboration), Phys. Rev. C 79, 034909 (2009), 0808.2041

[62] C. Adler, et al. (STAR Collaboration), Phys. Rev. Lett. 89, 092301 (2002), nucl-ex/0203016

[63] J. Adams, et al. (STAR Collaboration), Phys. Rev. Lett. 98, 062301 (2007), nucl-ex/0606014

[64] B. Abelev, et al. (STAR Collaboration), Phys. Rev. C 81, 024911 (2010), 0909.4131

[65] M. Aggarwal, et al. (STAR Collaboration), Phys. Rev. C 83, 024901 (2011), 1010.0142

[66] I. Arsene, et al. (BRAHMS Collaboration), Phys. Rev. C 72, 014908 (2005), nucl-ex/0503010

[67] S. Adler, et al. (PHENIX Collaboration), Phys. Rev. C 69, 034909 (2004), nucl-ex/0307022

[68] B. Abelev, et al. (ALICE Collaboration), Phys. Rev. Lett. 109, 252301 (2012), 1208.1974

[69] B. Abelev, et al. (ALICE Collaboration), Phys. Rev. C 88, 044910 (2013), 1303.0737 
[70] B. B. Abelev, et al. (ALICE Collaboration), Phys. Rev. Lett. 111, 222301 (2013), 1307.5530

[71] B. B. Abelev, et al. (ALICE Collaboration), Phys. Lett. B 728, 216 (2014), 1307.5543

[72] B. B. Abelev, et al. (ALICE Collaboration) (2014), 1404.0495

[73] M. Floris, talk at the "Quark Matter" conference (2014), http://qm2014.gsi.de

[74] P. Braun-Munzinger, K. Redlich, J. Stachel (2003), nucl-th/0304013

[75] A. Andronic, P. Braun-Munzinger, K. Redlich, et al., J. Phys. G 38, 124081 (2011), 1106.6321

[76] G. Chapline, A. Kerman, MIT-CTP-695 (1978)

[77] J. Cleymans, H. Oeschler, K. Redlich, Phys. Rev. C 59, 1663 (1999), nucl-th/9809027

[78] A. Andronic, P. Braun-Munzinger, J. Stachel, Phys. Lett. B 673, 142 (2009), 0812.1186

[79] J. Manninen, F. Becattini, Phys. Rev. C 78, 054901 (2008), 0806.4100

[80] M. Aggarwal, et al. (STAR Collaboration), Phys. Rev. C 83, 034910 (2011), 1008.3133

[81] R. Hagedorn, Lect. Notes Phys. 221, 53 (1985)

[82] R. Stock, Phys. Lett. B 456, 277 (1999), hep-ph/9905247

[83] P. Braun-Munzinger, J. Stachel, J. Phys. G 28, 1971 (2002), nucl-th/0112051

[84] P. Braun-Munzinger, J. Stachel, C. Wetterich, Phys. Lett. B 596, 61 (2004), nucl-th/0311005

[85] A. Andronic, D. Blaschke, P. Braun-Munzinger, et al., Nucl. Phys. A 837, 65 (2010), 0911.4806

[86] S. Floerchinger, C. Wetterich, Nucl. Phys. A 890-891, 11 (2012), 1202.1671

[87] J. Letessier, J. Rafelski, Eur. Phys. J. A 35, 221 (2008), nucl-th/0504028

[88] M. Petran, J. Rafelski, Phys. Rev. C 88, 2, 021901 (2013), 1303.0913

[89] A. Dumitru, L. Portugal, D. Zschiesche, Phys. Rev. C 73, 024902 (2006), nucl-th/0511084

[90] M. A. Stephanov, K. Rajagopal, E. V. Shuryak, Phys. Rev. Lett. 81, 4816 (1998), hep-ph/9806219

[91] A. Tawfik, Europhys. Lett. 75, 420 (2006), hep-ph/0410392 
[92] J. Cleymans, H. Oeschler, K. Redlich, et al., Phys. Rev. C 73, 034905 (2006), hep-ph/0511094

[93] A. Andronic, P. Braun-Munzinger, J. Stachel, et al., Phys. Lett. B 718, 80 (2012), 1201.0693

[94] M. Gaździcki, M. I. Gorenstein, Acta Phys. Polon. B 30, 2705 (1999), hep-ph/9803462

[95] A. Andronic, P. Braun-Munzinger, J. Stachel, et al., Phys. Lett. B 697, 203 (2011), 1010.2995

[96] R. Bellwied, S. Borsanyi, Z. Fodor, et al., Phys. Rev. Lett. 111, 202302 (2013), 1305.6297

[97] A. Bazavov, H. T. Ding, P. Hegde, et al., Phys. Rev. Lett. 111, 082301 (2013), 1304.7220

[98] F. Becattini, M. Bleicher, E. Grossi, et al. (2014), 1405.0710

[99] A. Bazavov, H. T. Ding, P. Hegde, et al. (2014), 1404.6511

[100] A. Bazavov, H. T. Ding, P. Hegde, et al. (2014), 1404.4043

[101] A. Andronic, F. Beutler, P. Braun-Munzinger, et al., Phys. Lett. B 675, 312 (2009), 0804.4132

[102] F. Becattini, P. Castorina, A. Milov, et al., Eur. Phys. J. C 66, 377 (2010), 0911.3026

[103] L. Adamczyk, et al. (STAR Collaboration) (2014), 1402.1558

[104] L. Adamczyk, et al. (STAR Collaboration), Phys. Rev. Lett. 112, 032302 (2014), 1309.5681

[105] M. A. Stephanov, K. Rajagopal, E. V. Shuryak, Phys. Rev. D 60, 114028 (1999), hep-ph/9903292

[106] A. Bazavov, H. T. Ding, P. Hegde, et al., Phys. Rev. Lett. 109, 192302 (2012), 1208.1220

[107] S. Borsanyi, Z. Fodor, S. Katz, et al. (2014), 1403.4576

[108] H. Gustafsson, H. Gutbrod, B. Kolb, et al., Phys. Rev. Lett. 52, 1590 (1984)

[109] E. Schnedermann, J. Sollfrank, U. W. Heinz, Phys. Rev. C 48, 2462 (1993), nucl-th/9307020

[110] M. Lisa, et al. (EOS Collaboration), Phys. Rev. Lett. 75, 2662 (1995), nucl-ex/9502001

[111] W. Reisdorf, et al. (FOPI Collaboration), Nucl. Phys. A 848, 366 (2010), 1005.3418

[112] C. Alt, et al. (NA49 Collaboration), Phys. Rev. C 77, 064908 (2008), 0709.4507 
[113] A. Andronic, et al. (FOPI Collaboration), Phys. Lett. B 612, 173 (2005), nucl-ex/0411024

[114] C. Pinkenburg, et al. (E895 Collaboration), Phys. Rev. Lett. 83, 1295 (1999), nucl-ex/9903010

[115] J. Barrette, et al. (E877 Collaboration), Phys. Rev. C 55, 1420 (1997), nucl-ex/9610006

[116] D. Adamova, et al. (CERES Collaboration), Nucl. Phys. A 698, 253 (2002)

[117] C. Alt, et al. (NA49 Collaboration), Phys. Rev. C 68, 034903 (2003), nucl-ex/0303001

[118] J. Adams, et al. (STAR Collaboration), Phys. Rev. C 72, 014904 (2005), nucl-ex/0409033

[119] B. Alver, et al. (PHOBOS Collaboration), Phys. Rev. Lett. 98, 242302 (2007), nucl-ex/0610037

[120] K. Aamodt, et al. (ALICE Collaboration), Phys. Rev. Lett. 105, 252302 (2010), 1011.3914

[121] S. Chatrchyan, et al. (CMS Collaboration), Phys. Rev. C 87, 014902 (2013), 1204.1409

[122] M. Demoulins, D. L'Hote, J. Alard, et al., Phys. Lett. B 241, 476 (1990)

[123] H. Gutbrod, B. Kolb, H. Schmidt, et al., Phys. Lett. B 216, 267 (1989)

[124] J.-Y. Ollitrault, Phys. Rev. D 46, 229 (1992)

[125] S. Adler, et al. (PHENIX Collaboration), Phys. Rev. Lett. 91, 182301 (2003), nucl-ex/0305013

[126] J. Adams, et al. (STAR Collaboration), Phys. Rev. Lett. 92, 052302 (2004), nucl-ex/0306007

[127] B. B. Abelev, et al. (ALICE Collaboration) (2014), 1405.4632

[128] H. Song, S. Bass, U. W. Heinz, Phys. Rev. C 89, 034919 (2014), 1311.0157

[129] B. Abelev, et al. (ALICE Collaboration), Phys. Rev. Lett. 111, 232302 (2013), 1306.4145

[130] L. Adamczyk, et al. (STAR Collaboration) (2014), 1401.3043

[131] K. Aamodt, et al. (ALICE Collaboration), Phys. Rev. Lett. 107, 032301 (2011), 1105.3865

[132] G. Aad, et al. (ATLAS Collaboration), JHEP 1311, 183 (2013), 1305.2942

[133] B. B. Abelev, et al. (ALICE Collaboration) (2014), 1406.2474

[134] B. Abelev, et al. (ALICE Collaboration), Phys. Lett. B 719, 29 (2013), 1212.2001 
[135] G. Aad, et al. (ATLAS Collaboration), Phys. Rev. Lett. 110, 182302 (2013), 1212.5198

[136] S. Chatrchyan, et al. (CMS Collaboration), Phys. Lett. B 724, 213 (2013), 1305.0609

[137] B. B. Abelev, et al. (ALICE Collaboration), Phys. Lett. B 726, 164 (2013), 1307.3237

[138] A. Adare, et al. (PHENIX Collaboration), Phys. Rev. Lett. 111, 212301 (2013), 1303.1794

[139] P. Bozek, W. Broniowski, Phys. Lett. B 718, 1557 (2013), 1211.0845

[140] K. Dusling, R. Venugopalan, Phys. Rev. Lett. 108, 262001 (2012), 1201.2658

[141] K. Dusling, R. Venugopalan, Phys. Rev. D 87, 094034 (2013), 1302.7018

[142] K. Werner, B. Guiot, I. Karpenko, et al., Phys. Rev. C 89, 064903 (2014),1312.1233

[143] G.-Y. Qin, B. Muller, Phys. Rev. C 89, 044902 (2014), 1306.3439

[144] A. Bzdak, B. Schenke, P. Tribedy, et al., Phys. Rev. C 87, 064906 (2013), 1304.3403

[145] P. Bozek, W. Broniowski, G. Torrieri, Phys. Rev. Lett. 111, 172303 (2013), 1307.5060

[146] M. A. Lisa, S. Pratt, R. Soltz, et al., Ann. Rev. Nucl. Part. Sci. 55, 357 (2005), nucl-ex/0505014

[147] K. Aamodt, et al. (ALICE Collaboration), Phys. Lett. B 696, 328 (2011), 1012.4035

[148] L. Adamczyk, et al. (STAR Collaboration) (2014), 1403.4972

[149] I. Karpenko, Y. Sinyukov, K. Werner, Phys. Rev. C 87, 024914 (2013), 1204.5351

[150] P. Bozek, W. Broniowski (2014), 1403.6042

[151] T. Schäfer, Physics 2, 88 (2009)

[152] H. Song, S. A. Bass, U. Heinz, et al., Phys. Rev. Lett. 106, 192301 (2011), 1011.2783

[153] E. Retinskaya, M. Luzum, J.-Y. Ollitrault, Phys. Rev. C 89, 014902 (2014), 1311.5339

[154] M. Luzum, P. Romatschke, Phys. Rev. C 78, 034915 (2008), 0804.4015

[155] F. G. Gardim, F. Grassi, M. Luzum, et al., Phys. Rev. Lett. 109, 202302 (2012), 1203.2882

[156] P. Kovtun, D. T. Son, A. O. Starinets, Phys. Rev. Lett. 94, 111601 (2005), hep-th/0405231 
[157] P. Danielewicz, Phys. Lett. B 146, 168 (1984)

[158] W. Schmidt, U. Katscher, B. Waldhauser, et al., Phys. Rev. C 47, 2782 (1993)

[159] N. Auerbach, S. Shlomo, Phys. Rev. Lett. 103, 172501 (2009), 0908.4441

[160] J. Berges, J.-P. Blaizot, F. Gelis, J. Phys. G 39, 085115 (2012), 1203.2042

[161] A. Kurkela, E. Lu (2014), 1405.6318

[162] K. Kajantie, H. Miettinen, Z. Phys. C 9, 341 (1981)

[163] C. Gale, Nucl. Phys. A 910-911, 147 (2013), 1208.2289

[164] R. Rapp, Adv. High Energy Phys. 2013, 148253 (2013), 1304.2309

[165] R. Rapp, J. Wambach, H. van Hees, Landolt-Börnstein I/23 (2010), 0901.3289

[166] A. Adare, et al. (PHENIX Collaboration), Phys. Rev. Lett. 104, 132301 (2010), 0804.4168

[167] C. Gale, Physics 3, 28 (2010), URLhttp://link.aps.org/doi/10.1103/Physics.3.28

[168] M. Wilde (ALICE Collaboration), Nucl. Phys. A 904-905, 573c (2013), 1210.5958

[169] A. Adare, et al. (PHENIX Collaboration), Phys. Rev. Lett. 109, 122302 (2012), 1105.4126

[170] D. Lohner (ALICE Collaboration), J. Phys. Conf. Ser. 446, 012028 (2013), 1212.3995

[171] H. van Hees, C. Gale, R. Rapp, Phys. Rev. C 84, 054906 (2011), 1108.2131

[172] C. Shen, U. W. Heinz, J.-F. Paquet, et al., Phys. Rev. C 89, 044910 (2014), 1308.2440

[173] D. d'Enterria, Landolt-Börnstein I/23 (2010), 0902.2011

[174] R. Averbeck, Prog. Part. Nucl. Phys. 70, 159 (2013)

[175] T. Matsui, H. Satz, Phys. Lett. B 178, 416 (1986)

[176] J. Bjorken, Preprint FERMILAB-PUB-82-059-THY (1982)

[177] K. Adcox, et al. (PHENIX Collaboration), Phys. Rev. Lett. 88, 022301 (2002), nucl-ex/0109003

[178] C. Adler, et al. (STAR Collaboration), Phys. Rev. Lett. 89, 202301 (2002), nucl-ex/0206011

[179] K. Aamodt, et al. (ALICE Collaboration), Phys. Lett. B 696, 30 (2011), 1012.1004

[180] S. Chatrchyan, et al. (CMS Collaboration), Eur. Phys. J. C 72, 1945 (2012), 1202.2554

[181] B. Abelev, et al. (ALICE Collaboration), Phys. Lett. B 720, 52 (2013), 1208.2711 
[182] S. Chatrchyan, et al. (CMS Collaboration), JHEP 1210, 087 (2012), 1205.5872

[183] G. Aad, et al. (ATLAS Collaboration), Phys. Lett. B 719, 220 (2013), 1208.1967

[184] B. Abelev, et al. (ALICE Collaboration), Phys. Rev. Lett. 110, 082302 (2013), 1210.4520

[185] B. B. Abelev, et al. (ALICE Collaboration) (2014), 1405.2737

[186] S. Chatrchyan, et al. (CMS Collaboration), Phys. Lett. B 710, 256 (2012), 1201.3093

[187] S. Chatrchyan, et al. (CMS Collaboration), Phys. Rev. Lett. 106, 212301 (2011), 1102.5435

[188] S. Chatrchyan, et al. (CMS Collaboration), Phys. Lett. B 715, 66 (2012), 1205.6334

[189] T. Renk, Nucl. Phys. A 910-911, 43 (2013), 1207.4885

[190] M. Panero, K. Rummukainen, A. Schäfer, Phys. Rev. Lett. 112, 162001 (2014), 1307.5850

[191] K. M. Burke, A. Buzzatti, N. Chang, et al. (2013), 1312.5003

[192] B. Abelev, et al. (ALICE Collaboration), JHEP 1209, 112 (2012), 1203.2160

[193] $\mathrm{J} / \psi$ results from $C M S$ in $\mathrm{PbPb}$ collisions, with $150 \mu b^{-1}$ data, Tech. Rep. CMSPAS-HIN-12-014, CERN, Geneva (2012)

[194] Y. L. Dokshitzer, D. Kharzeev, Phys. Lett. B 519, 199 (2001), hep-ph/0106202

[195] A. Adare, et al. (PHENIX Collaboration), Phys. Rev. C 84, 044905 (2011), 1005.1627

[196] A. Adare, et al. (PHENIX Collaboration), Phys. Rev. Lett. 98, 172301 (2007), nucl-ex/0611018

[197] L. Adamczyk, et al. (STAR Collaboration) (2014), 1404.6185

[198] B. B. Abelev, et al. (ALICE Collaboration) (2014), 1405.2001

[199] L. Adamczyk, et al. (STAR Collaboration) (2014), 1405.6348

[200] M. He, R. J. Fries, R. Rapp (2014), 1401.3817

[201] S. Chatrchyan, et al. (CMS Collaboration), Phys. Lett. B 730, 243 (2014), 1310.0878

[202] B. Abelev, et al. (ALICE Collaboration), JHEP 1403, 013 (2014), 1311.0633

[203] S. Chatrchyan, et al. (CMS Collaboration) (2013), 1312.4198

[204] F. Karsch, H. Satz, Z. Phys. C 51, 209 (1991)

[205] S. Digal, P. Petreczky, H. Satz, Phys. Rev. D 64, 094015 (2001), hep-ph/0106017 
[206] F. Karsch, D. Kharzeev, H. Satz, Phys. Lett. B 637, 75 (2006), hep-ph/0512239

[207] L. Kluberg, H. Satz, Landolt-Börnstein I/23 (2010), 0901.3831

[208] M. Asakawa, T. Hatsuda, Phys. Rev. Lett. 92, 012001 (2004), hep-lat/0308034

[209] A. Mocsy, P. Petreczky, Phys. Rev. D 77, 014501 (2008), 0705.2559

[210] A. Mocsy, P. Petreczky, Phys. Rev. Lett. 99, 211602 (2007), 0706.2183

[211] K. Morita, S. H. Lee, Phys. Rev. C 85, 044917 (2012), 1012.3110

[212] H. Ding, A. Francis, O. Kaczmarek, et al., Phys. Rev. D 86, 014509 (2012), 1204.4945

[213] S. H. Lee, K. Morita, T. Song, et al., Phys. Rev. D 89, 094015 (2014), 1304.4092

[214] T. Hayata, K. Nawa, T. Hatsuda, Phys. Rev. D 87, 101901 (2013), 1211.4942

[215] A. Rothkopf, JHEP 1404, 085 (2014), 1312.3246

[216] S. Borsanyi, S. Dürr, Z. Fodor, et al., JHEP 1404, 132 (2014), 1401.5940

[217] A. Mocsy, P. Petreczky, M. Strickland, Int. J. Mod. Phys. A 28, 1340012 (2013), 1302.2180

[218] P. Braun-Munzinger, J. Stachel, Phys. Lett. B 490, 196 (2000), nucl-th/0007059

[219] A. Andronic, P. Braun-Munzinger, K. Redlich, et al., Nucl. Phys. A 789, 334 (2007), nucl-th/0611023

[220] P. Braun-Munzinger, J. Stachel, Landolt-Börnstein I/23 (2010), 0901.2500

[221] R. L. Thews, M. Schroedter, J. Rafelski, Phys. Rev. C 63, 054905 (2001), hep-ph/0007323

[222] Y.-P. Liu, Z. Qu, N. Xu, et al., Phys. Lett. B 678, 72 (2009), 0901.2757

[223] X. Zhao, R. Rapp, Nucl. Phys. A 859, 114 (2011), 1102.2194

[224] A. Emerick, X. Zhao, R. Rapp, Eur. Phys. J. A 48, 72 (2012), 1111.6537

[225] K. Zhou, N. Xu, Z. Xu, et al., Phys. Rev. C 89, 054911 (2014), 1401.5845

[226] A. Adare, et al. (PHENIX Collaboration), Phys. Rev. Lett. 98, 232301 (2007), nucl-ex/0611020

[227] B. B. Abelev, et al. (ALICE Collaboration), Phys. Lett. B 743, 314 (2014), 1311.0214

[228] S. Chatrchyan, et al. (CMS Collaboration), JHEP 1205, 063 (2012), 1201.5069

[229] X. Zhao, A. Emerick, R. Rapp, Nucl. Phys. A 904-905, 611c (2013), 1210.6583

[230] B. Abelev, et al. (ALICE Collaboration), Phys. Rev. Lett. 109, 072301 (2012), 1202.1383 
[231] E. Abbas, et al. (ALICE Collaboration), Phys. Rev. Lett. 111, 162301 (2013), 1303.5880

[232] L. Adamczyk, et al. (STAR Collaboration), Phys. Rev. Lett. 111, 5, 052301 (2013), 1212.3304

[233] F. Prino, et al. (NA50 Collaboration), Eur. Phys. J. C 61, 853 (2009), 0906.5376

[234] H. Liu, K. Rajagopal, U. A. Wiedemann, Phys. Rev. Lett. 98, 182301 (2007), hep-ph/0607062

[235] M. Laine (2011), 1108.5965

[236] B. B. Abelev, et al. (ALICE Collaboration), JHEP 1402, 073 (2014), 1308.6726

[237] R. Aaij, et al. (LHCb Collaboration), JHEP 1402, 072 (2014), 1308.6729

[238] A. Adare, et al. (PHENIX Collaboration), Phys. Rev. Lett. 111, 20, 202301 (2013), 1305.5516

[239] B. B. Abelev, et al. (ALICE Collaboration) (2014), 1405.3796

[240] S. Chatrchyan, et al. (CMS Collaboration), JHEP 04, 103 (2014), 1312.6300

[241] S. Chatrchyan, et al. (CMS Collaboration), Phys. Rev. Lett. 109, 222301 (2012), 1208.2826

[242] B. B. Abelev, et al. (ALICE Collaboration) (2014), 1405.4493

[243] A. Adare, et al. (PHENIX Collaboration) (2014), 1404.2246

[244] M. Strickland, AIP Conf. Proc. 1520, 179 (2013), 1207.5327

[245] A. Andronic, F. Beutler, P. Braun-Munzinger, et al., Phys. Lett. B 678, 350 (2009), 0904.1368 